\begin{tabular}{|c|c|c|}
\hline $\begin{array}{c}\text { 2. To: (Receiving organization) } \\
\text { Distribution }\end{array}$ & $\begin{array}{l}\text { 3. From: (originating organization) } \\
\text { NS\&L Safety Analysis }\end{array}$ & $\begin{array}{r}\text { 4. Related EDT No.: } \\
\text { N/A }\end{array}$ \\
\hline $\begin{array}{l}\text { 5. Proj./Prog./Dept./Div: } \\
\text { River Protection Project }\end{array}$ & $\begin{array}{l}\text { 6. Design Authority/ Design Agent/Cog. } \\
\text { Engr:: }\end{array}$ & $\begin{array}{r}\text { 7. Purchase Order No.: } \\
\text { N/A }\end{array}$ \\
\hline \multirow{2}{*}{\multicolumn{2}{|c|}{$\begin{array}{l}\text { 8. Originator Remarks: } \\
\text { RPP-5453 is transmitted for your review and approval }\end{array}$}} & $\begin{array}{r}\text { 9. Equip./Component No.: } \\
\qquad \mathrm{N} / \mathrm{A}\end{array}$ \\
\hline & & $\begin{array}{r}\text { 10. System/Bldg./Facility: } \\
\text { N/A }\end{array}$ \\
\hline \multirow[t]{3}{*}{ 11. Receiver Remarks: } & \multirow[t]{3}{*}{ Basel ine Document? $[\cdot]$ Yes $[\mathrm{X}]$ No } & $\begin{array}{r}\text { 12. Ma.jor Assm. Dwg. No,: } \\
\text { N/A }\end{array}$ \\
\hline & & $\begin{array}{l}\text { 13. Permit/Permit Applicat ion No.: } \\
\text { N/A }\end{array}$ \\
\hline & & 14. Required Response Date: \\
\hline
\end{tabular}

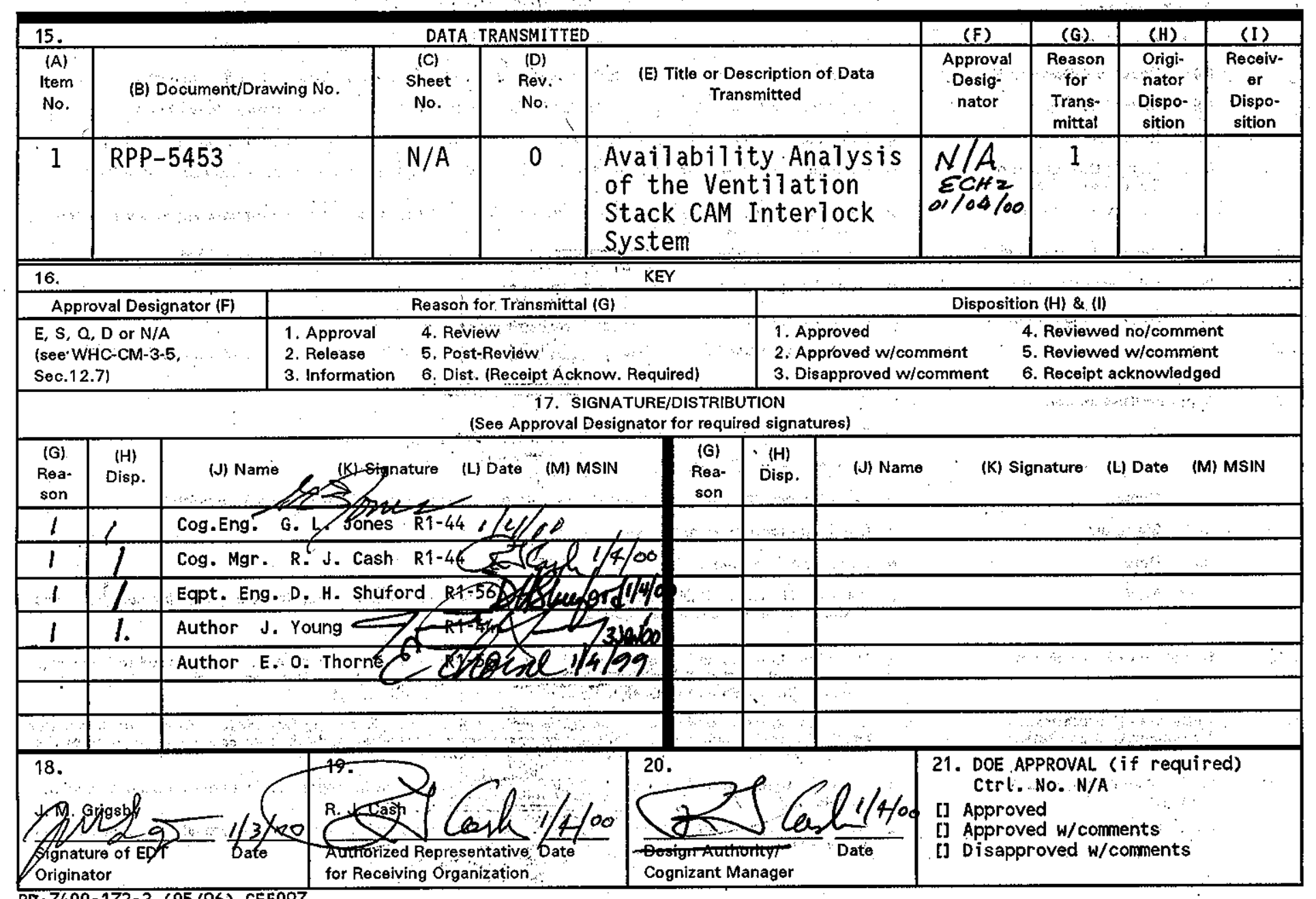




\section{Availability Analysis of the Ventilation Stack CAM Interlock System}

\section{J. Young}

Lockheed Martin Hanford Corporation

Richland, WA 99352

U.S. Department of Energy Contract DE-AC06-99RL14047

$\begin{array}{ll}\text { EDT/ECN: } 628638 & \text { UC: } 2030 \\ \text { Org Code: } 74 \text { F00 } & \text { Charge Code: } 109310 / \text { BA10 } \\ \text { B\&R Code: N/A } & \text { Total Pages: } 38\end{array}$

Key Words: Authorization Basis, AB, Availability, Continuous Air Monitor, CAM, Interlock, Surveillance, System,

Abstract: Ventilation Stack Continuous Air Monitor (CAM) Interlock System failure modes, failure frequencies and system availability have been evaluated for the RPP. The evaluation concludes that CAM availability is as high as assumed in the safety analysis and that the current routine system surveillance is adequate to maintain this availability. Further, requiring an alarm to actuate upon CAM failure is not necessary to maintain the availability credited in the safety analysis, nor is such an arrangement predicted to significantly improve system availability. However, if CAM failures were only detected by the 92-day functional tests required in the Authorization Basis (AB), CAM availability would be much less than that credited in the safety analysis. Therefore it is recommended that the current surveillance practice of daily simple system checks, 30-day source checks and 92-day functional tests be continued in order to maintain CAM availability.

TRADEMARK DISCLAIMER. Reference herein to any specific commercial product, process, or service by trade name, trademark, manufacturer, or otherwise, does not necessarily constitute or imply its endorsement, recommendation, or favoring by the United States Government or any agency thereof or its contractors or subcontractors.

Printed in the United States of America. To obtain copies of this document, contact: Document Control Services, P.0. Box 950, Mailstop H6-08, Richland WA 99352, Phone (509) 372-2420; Fax (509) 376-4989.
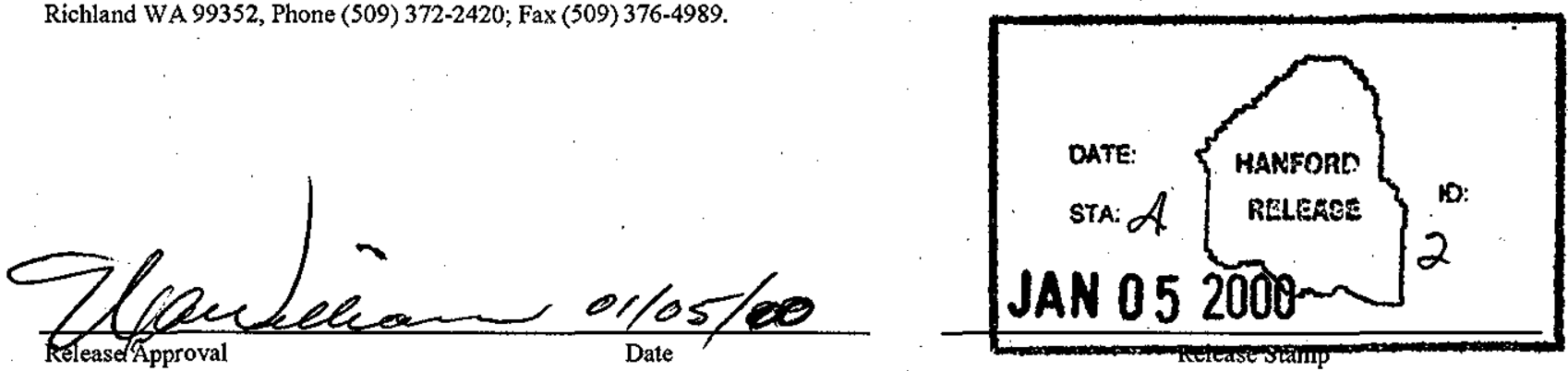

Approved for Public Release 


\title{
Availability Analysis of the Ventilation Stack CAM Interlock System
}

\author{
J. M. Grigsby
}

G\&P Consulting, Inc.

E. O. Thorne

Tekton Resources, Inc.

J. Young

MSI

Date Published

January 2000

Prepared for the U.S. Department of Energy

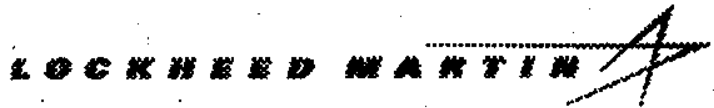

P.O. Box 1500

Richland, Washington

Hanford Management and Integration Contractor for the

U.S. Department of Energy under Contract DE-ACO6-99RL14047 
RPP-5453 REV 0

This page intentionally left blank. 


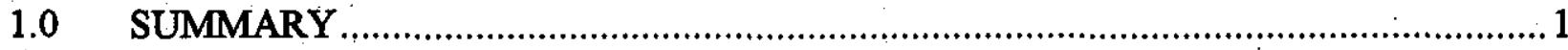

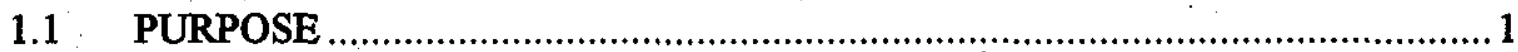

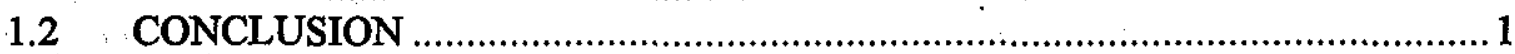

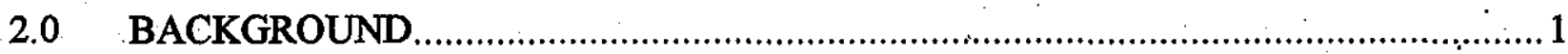

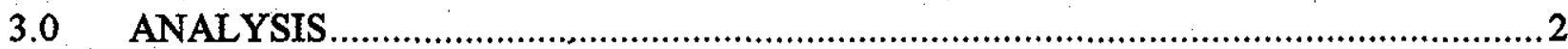

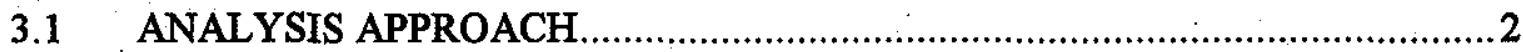

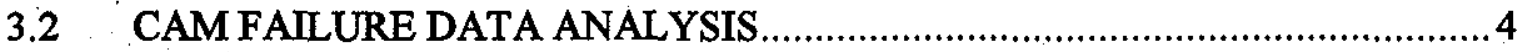

$3.3 \quad$ AVAILABILITY ANALYSIS RESULTS …................................................

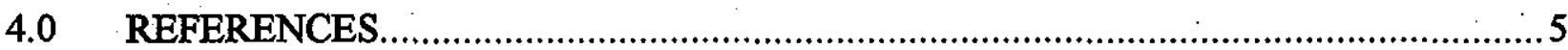

\section{APPENDTXES}

A SUMMARY: HOURS OF SERVICE AND FREQUENCY OF CONTINUOUS AIR MONITOR FAILURES .............................................................

B CONTINUOUS AIR MONITOR AVAILABILITY ANALYSIS .........................................

C AVAILABILITY ANALYSIS CASES FOR RPP CONTINUOUS AIR MONITOR INTERLOCK SYSTEMS

D COPY OF CONTRACTOR SURVEILLANCE REPORT RESPONSE S99-TOD-TF-042-FO1

E CHECKLIST FOR TECHNICAL PEER REVIEW E-1

\section{LIST OF TABLES}

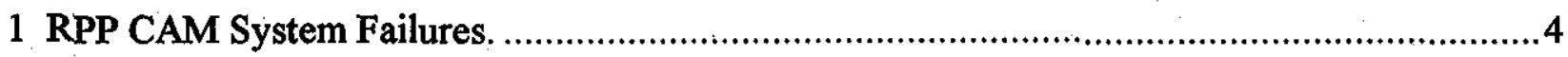

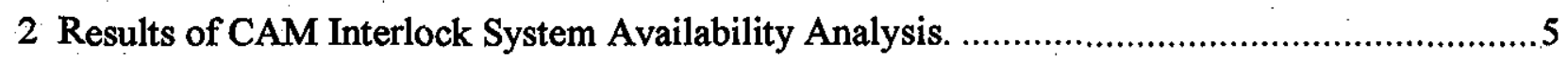




\section{LIST OF TERMS}

\begin{tabular}{|c|c|}
\hline $\mathrm{AB}$ & authorization basis \\
\hline CAM & continuous air monitor \\
\hline FSAR & Final Safety Analysis Report \\
\hline HEPA & High Efficiency Particulate Air (filter) \\
\hline MTBF & mean time between failures \\
\hline MTTR & mean time to repair \\
\hline RAM & reliability, availability, and maintainability \\
\hline RPP & River Protection Project \\
\hline TSR & Technical Safety Requirement \\
\hline
\end{tabular}




\subsection{SUMMARY}

\subsection{PURPOSE}

The purpose of this report is to perform an availability analysis of the River Protection Project (RPP) Ventilation Stack Continuous Air Monitor (CAM) Interlock System to support development of responses to Contractor Surveillance Report Response Number: S99-TOD-TF-042-F01 (included in Appendix D) items 2 and 3.

- Item 2: Perform an engineering evaluation to determine failure modes that affect the intended safety function of the CAMs with regard to the interlock TSR and make necessary recommendations to correct any deficiencies.

- Item 3: Analyze surveillance frequency to determine adequacy.

The analysis computes CAM availability based on current RPP surveillance and maintenance practice, with a 30 minute alarm of all CAM failures, and assuming the TSR surveillance is the only CAM surveillance conducted.

\subsection{CONCLUSION}

Ventilation Stack Continuous Air Monitor Interlock System failure modes, failure frequencies and system availability have been evaluated for the RPP. The evaluation concludes that CAM availability is as high as assumed in the safety analysis and that the current routine system surveillance is adequate to maintain this availability. Further, requiring an alarm to actuate upon CAM failure is not necessary to maintain the availability credited in the safety analysis, nor is such an arrangement predicted to significantly improve system availability. However, if CAM failures were only detected by the 92-day functional tests required in the Authorization Basis (AB), CAM availability would be much less than that credited in the safety analysis. Therefore it is recommended that the current surveillance practice of daily simple system checks, 30-day source checks and 92-day functional tests be continued in order to maintain CAM availability.

\subsection{BACKGROUND}

The safety function of the CAM Interlock System is to shutdown active exhaust ventilation on a high radiation reading to limit the release of airborne radionuclides after HEPA filters are damaged by an in-tank spray leak or HEPA failure due to a high temperature accident. The HEPA Filter Failure - Exposure to High Temperature accident analysis (Tank Waste Remediation System Final Safety Analysis Report [FSAR] Section 3.3.2.4.2) assumes that the interlock shuts down the ventilation flow within 10 minutes of detecting the high radiation condition. No explicit CAM Interlock System availability is stated in the safety analysis, however the assumed availability can be inferred from the analysis in FSAR Section 3.4.3.1.1, 
Failure of Controls for HEPA Filter Failure - Exposure to High Temperature or Pressure. This analysis states that frequency of the initiating event (HEPA failure due to high temperature) is "anticipated" ( $>10^{-2} / \mathrm{yr}$ to $\left.\leq 10^{\%} / \mathrm{yr}\right)$, while the frequency of the accident with failed controls (CAM Interlocks) is "unlikely" ( $>10^{-4} / \mathrm{yr} \leq 10^{-2} / \mathrm{yr}$ ). This is nomithally a factor of 100 reduction in the frequency, implying that the CAM Interlock system has a $99 \%$ probability of being available to mitigate this accident. The safety analysis assumption for CAM Interlock system availability is thus 0.99 .

The question raised in Surveillance Number: S99-TOD-TF-042-F01 is, "can the CAM Interlock System meet its operability requirements if CAM Interlock System failures are not alarmed at a continuously manned (e.g., a control room manned 24 hours a day) location?" The CAM failure mode evaluation and-surveillance frequency analysis question is clarified for this analysis to be, "Given the CAM Interlock System failure history, is it necessary to alarm CAM Interlock System failures at a continuously manned location in order to maintain the system availability credited in the safety analysis?"

This question is addressed by evaluating CAM Interlock System failure modes and failure rates over the past two years with system availability analysis and comparing the system availability to that credited in the safety analysis.

\subsection{ANALYSIS}

\subsection{ANALYSIS APPROACH}

The current RPP AB identifies the Continuous Air Monitor Interlock Systems as safety controls. The assessment of the effectiveness of the CAMs was based on the qualitative estimate that the CAMs reduced the accident frequency by about two orders of magnitude. This implies that the CAMs have an availability of 0.99 . Availability is the probability that a system is in an operable condition at any random point in time.

An analysis was done to determine how accurate the qualitative assessment was and to identify what surveillance is required to achieve a CAM availability of 0.99 . The analysis was based on two years of experience at RPP and the application of standard computational methods currently in use in system reliability, availability, and maintainability (RAM) analysis and for Probabilistic Risk Assessments (NUREG/CR-2300).

Availability can be computed by the following equation, where failures are discovered as a result of a surveillance activity (NUREG/CR-2300):

$$
A=1-1 / 2 \lambda t
$$

where:

A: system/equipment availability

$\lambda$ : system/equipment failure rate (failures/hour) 
t: surveillance interval (hours)

Alternatively, if there is relatively instantaneous failure detection, availability can be represented by (NUREG/CR-2300):

$$
A=M T B F /(M T B F+M T T R)
$$

where:

MTBF: system/equipment mean time between failure (1/ $\lambda$ )

MTTR: system/equipment mean time to repair (repair time includes failure detection and correction times)

Based on current RPP practice, equation (1) and equation (2) were both used to assess the current availability of the CAMs, and to determine if availability is driven more by the surveillance frequency or by the time needed to repair the system. CAM failure history was used to derive CAM failure rates. Because the CAMs are currently subject to three different types of surveillance actions, the CAM system was addressed as consisting of three subsystems, each made up of those components whose failure could be detected by a particular surveillance activity.

1.: Equipment whose failures are detectable by the 24-hour simple system check surveillance;

2. Additional equipment whose failures are detectable by the 30-day source check surveillance; and

3. The remaining equipment whose failures are detectable by the 3-month Technical Safety Requirements (TSR) functional test surveillance.

Based on defining the CAM Interlock System as made up of three elements, the availability of the CAMs is computed as follows:

$$
A_{C A M}=\left(A_{1}\right)\left(A_{2}\right)\left(A_{3}\right)
$$

In practice, a number of different system checks are performed such as 6-hour operations checks, 24-hour Health Physics Technician checks, 15-day filter changes, etc. Some failures are alarmed. For the purposes of this availability analysis, alarmed failures and failures found during routine 6-hour operations checks are assumed to only be detected by the 24-hour (daily) simple system checks. Failures actually detected during 15-day filter changes are assumed to only be detected by the 30-day source checks.

Because the data available often does not include the exact time that the failure occurred, it has been assumed that failures occur, on average, in the middle of the surveillance interval. Sensitivity studies were performed to examine the impact of various proposed surveillance alternatives on CAM availability based on current failure experience. The cases examined were 
(1) current practices, (2) reliance on the 92-day TSR functional test only, and (3) if CAM failures could be automatically alarmed and thus be instantaneously detected. In the last case, it was assumed that the alarm would detect failures for all surveillance activities listed previously. This assumption that the alarm could detect all of the failures detected by all hour surveillance activity is probably optimistic.

\subsection{CAM FAILURE DATA ANALYSIS}

Operational data from October 1997 through September 1999 were gathered and evaluated for 40.CAM Systems in operation at RPP whose failures are tracked in the occurrence reporting system. This operating experience comprises over 700,800 CAM hours of operation. System failures experienced over this time period are detailed in Appendix A. The failure modes and the surveillance that detected the failure have been evaluated. The results from the evaluation of operating experience are summarized in Table 1. The mean time between failures (MTBF) for the CAM System is 8,870 hours while the observed availability is $0.995(99.5 \%)$. This is based on excluding exhauster down time when CAMs are not required. Based on this calculation, the observed availability is greater than the availability assumed in the safety analysis.

Table 1. RPP CAM System Failures.

\begin{tabular}{|l|c|c|c|c|}
\hline & $\begin{array}{c}\text { Found by Daily } \\
\text { System Check }\end{array}$ & $\begin{array}{c}\text { Found by } \\
\text { Source Check }\end{array}$ & $\begin{array}{c}\text { Found by } \\
\text { Functional Test }\end{array}$ & Total \\
\hline Number of failures & 70 & 9 & 0 & 79 \\
\hline $\begin{array}{l}\text { Failure frequency } \\
(\lambda)\end{array}$ & $9.99 \mathrm{E}-5 / \mathrm{hr}$ & $1.28 \mathrm{E}-5 / \mathrm{hr}$ & $1.43 \mathrm{E}-6^{\mathrm{c}}$ & $1.1 \mathrm{E}-4 / \mathrm{hr}$ \\
\hline MTBF (1/ $\lambda)$ & $10,000 \mathrm{hrs}$ & $78,000 \mathrm{hrs}$ & - & $8,870 \mathrm{hrs}$ \\
\hline MTTR $^{\mathrm{a}}$ & $11 \mathrm{hrs}$ & $295 \mathrm{hrs}$ & - & $43.5 \mathrm{hrs}$ \\
\hline Availability & - & - & - & $\mathbf{0 . 9 9 5}^{\mathrm{b}}$ \\
\hline
\end{tabular}

${ }^{a}$ Excludes the time when exhauster was shutdown (thus CAM was not required to be available).

${ }^{b}$ Fraction of the past two years that the CAMs have been available based on operating data.

${ }^{\circ}$ Conservatively assumes one failure.

\subsection{AVAILABLITY ANALYSIS RESULTS}

The availability analysis equations described above have been applied to the CAM operating data to study how system availability may be affected by changes in surveillance practices. The calculations for the specific cases analyzed are included in Appendix B, including inputs and outputs for each case. The results are summarized in Table 2.

As shown in Table 2, availability analysis predicts that the current practice of performing a daily simple system check, a 30-day source check, and a 92-day system functional test results in an availability of 0.993 . This compares well with the observed availability of 0.995 shown in Table 1. Further, having CAM Interlock System failures alarm at a continuously manned 


\section{RPP-5453 REV 0}

location is not necessary to maintain availability greater than 0.99 . Such an arrangement is not predicted to significantly improve system availability because the system availability is driven

more by repair time than detection time. Finally, it is noted that most CAM failures are detected by the daily simple checks while no reported failures have been detected by the TSR functional tests. Relying only on the 92-day TSR surveillance is predicted to reduce system availability to 0.876 .

Table 2. Results of CAM Interlock System Availability Analysis.

\begin{tabular}{|l|c|c|c|c|}
\hline \multicolumn{1}{|c|}{ Case } & $\begin{array}{c}\text { Simple System } \\
\text { Check Frequency } \\
\text { (hrs) }\end{array}$ & $\begin{array}{c}\text { Source Check } \\
\text { Frequency } \\
\text { (days) }\end{array}$ & $\begin{array}{c}\text { System Functional } \\
\text { Test Frequency } \\
\text { (days) }\end{array}$ & $\begin{array}{c}\text { Calculated } \\
\text { CAMS } \\
\text { Availability }\end{array}$ \\
\hline $\begin{array}{l}\text { Case 1 -Current } \\
\text { Practice }\end{array}$ & 24 & 30 & 92 & 0.993 \\
\hline $\begin{array}{l}\text { Case 2- } \\
\text { Immediate } \\
\text { Response Based } \\
\text { on Alarmed } \\
\text { Failures }\end{array}$ & $0.5^{*}$ & $0.5^{*}$ & $0.5^{*}$ & 0.995 \\
\hline $\begin{array}{l}\text { Case 3-TSR } \\
\text { Surveillance Only }\end{array}$ & None & None & 92 (TSR) & 0.876 \\
\hline
\end{tabular}
failures.

\subsection{REFERENCES}

Contractor Surveillance Report Response, "Technical Safety Requirements," Number S99-TOD-TF-042-FO1, B. J. Harp, U.S. Department of Energy, Richland Operation Office (DOE-RL), September 2, 1999.

FSAR, 1999, Tank Waste Remediation System Final Safety Analysis Report,

- HNF-SD-WM-SAR-067, Revision 1, Lockheed Martin Hanford Company, Richland, Washington.

NUREG/CR-2300, 1983, PRA (Probabilistic Risk Assessments) Procedures Guide: A Guide to the Performance of Probabilistic Risk Assessments for Nuclear Power Plants, American Nuclear Society, LaGrange Park, Illinois. 
RPP-5453 REV 0

This page intentionally left blank. 
RPP-5453 REV 0

\section{APPENDIX A}

SUMMARY: HOURS OF SERVICE AND FREQUENCY OF CONTINUOUS AIR MONITOR FAILURES 
RPP-5453 REV 0

This page intentionally left blank. 


\section{SUMMARY: HOURS OF SERVICE AND FREQUENCY OF CONTINUOUS AIR MONITOR FAILURES \\ (see Table A-1)}

1. Time Unavailable begins with the time the incident was discovered; and ends with the completion of repairs, or shutdown of the fan.

2. Hours Unavailable is the Time Unavailable plus one half the surveillance interval when the actual failure time is unknown.

3. There are 40 Beta-Gamma Continuous Air Monitor locations used in this evaluation.

4. The evaluation starts with Occurrence Reports from October of 1997 and ends with reports from September 1999.

5. In cases where the date of repair is known, but the time is not available, the assumed completion time is taken as $\mathbf{3 0}$ minutes before the end of the next shift or midnight of that day.

6. Discovery Period indicates the method and periodicity of failure discovery.

Total CAM hours, $10 / 97$ through 9/99: $\quad 700800$

Occurrence Reports:

CAM incidents:

CAM hours unavailable:
58

87 (including 8 incidents not involving a CAM failure)

Means of failure discovery:

$\begin{array}{lr}\text { Ops }(6 \mathrm{hr}) & 31 \\ \text { HPT }(24 \mathrm{hr}) & 14 \\ \text { Craft }(0 \mathrm{hr}) & 6 \\ \text { Cont Rm Ops }(0 \mathrm{hr}) & 15 \\ \text { Testing }(0 \mathrm{hr}) & 4 \\ \text { Source Chk }(720 \mathrm{hr}) & 5 \\ \text { Filter Chg }(336 \mathrm{hr}) & 4\end{array}$

Discovery Totals

$\leq 24 \mathrm{hrs}$

$\leq 720>24 \mathrm{hrs}$

$>720 \mathrm{hrs}$

31

14

6 4 5 4 
RPP-5453 REV 0

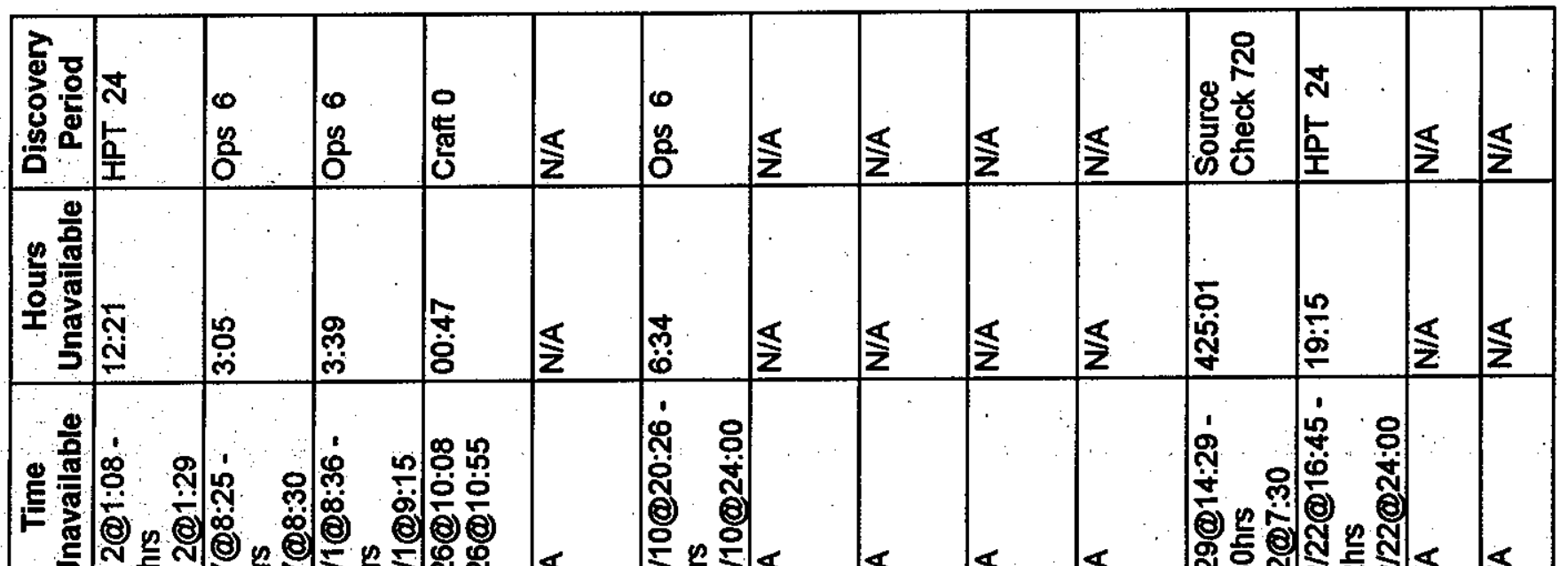

曾

5 든

$\begin{array}{llllllll} & 0\end{array}$

索

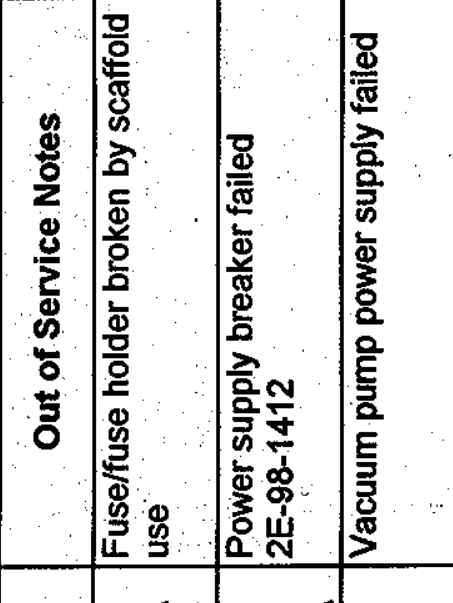

올

8

递



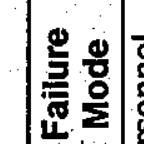

$=$ f $=$

迹

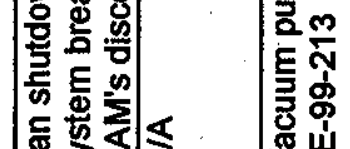
कूष $>$ 虫 站

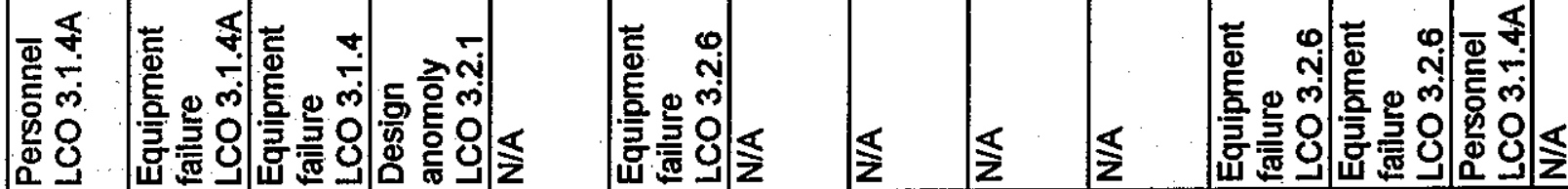

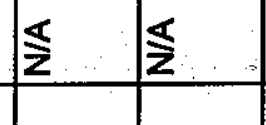

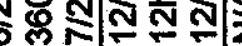

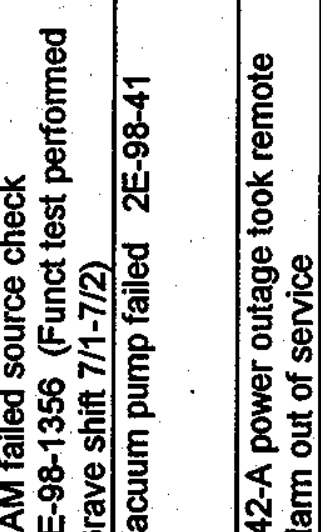

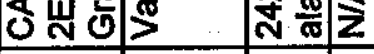

흥

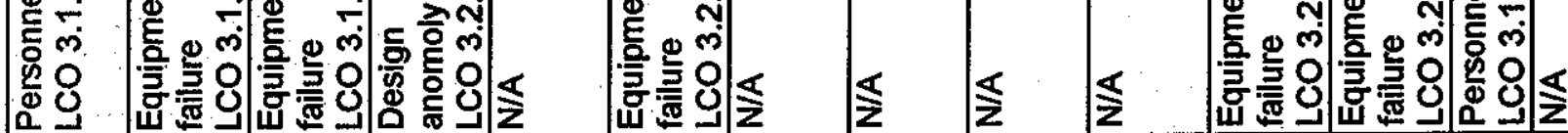

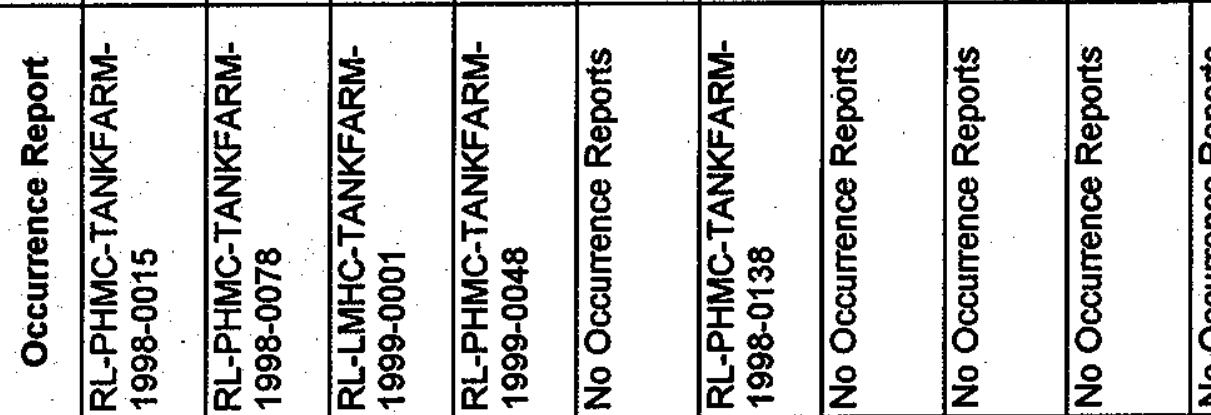

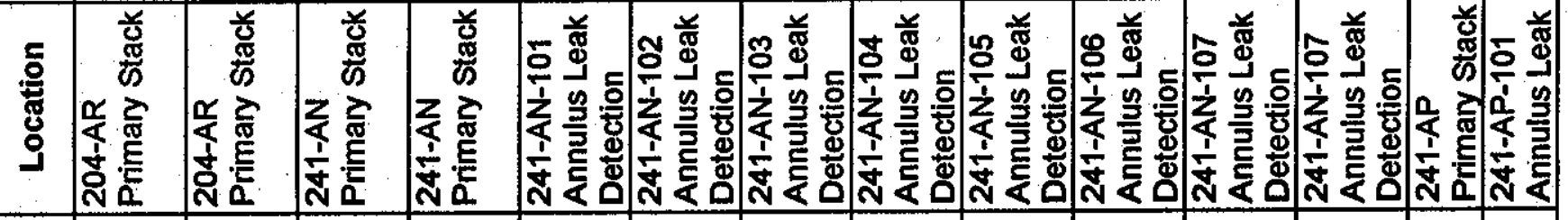
o m $\checkmark$ 


\begin{tabular}{|c|c|c|c|c|c|c|c|c|c|c|c|c|c|}
\hline 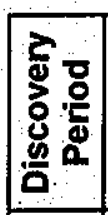 & $\underline{\mathbf{s}}$ & $\underline{\mathbf{z}}$ & $\underline{\Sigma}$ & 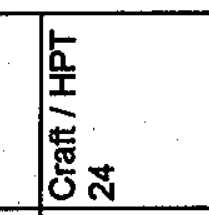 & 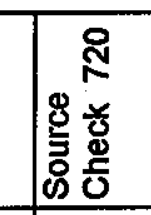 & $\underline{\mathbf{s}}$ & $\underline{\mathbf{z}}$ & $\underline{\mathbf{s}}$ & 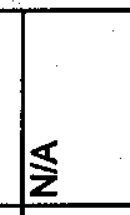 & \begin{tabular}{|l}
0 \\
0 \\
0 \\
0
\end{tabular} & 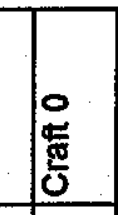 & \begin{tabular}{|l}
0 \\
0 \\
$\vdots$ \\
0 \\
0
\end{tabular} & \begin{tabular}{|l}
0 \\
0 \\
0 \\
0 \\
0
\end{tabular} \\
\hline $\begin{array}{r}0.5 \\
5 \\
5\end{array}$ & $\underline{\mathbf{s}}$ & $\underline{\mathbf{s}}$ & $\underline{\mathbf{z}}$ & 量 & 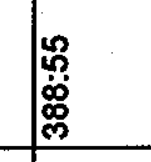 & $\underline{z}$ & $\underline{\Sigma}$ & $\underline{\mathbf{z}}$ & 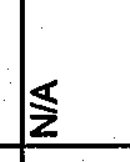 & 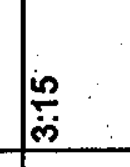 & & 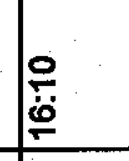 & $\stackrel{n}{0.0}$ \\
\hline $\begin{array}{r}\frac{0}{0} \\
\frac{5}{5} \\
5 \\
5 \\
5\end{array}$ & $\Sigma$ & $\underline{\mathbf{z}}$ & 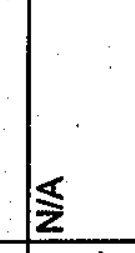 & 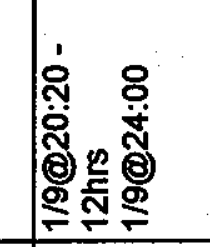 & 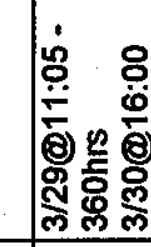 & & $\underline{\mathbf{z}}$ & $\underline{\mathbf{z}}$ & $\$$ & 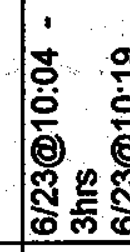 & 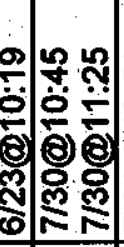 & : & 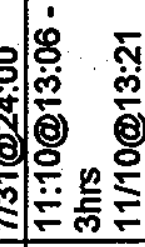 \\
\hline 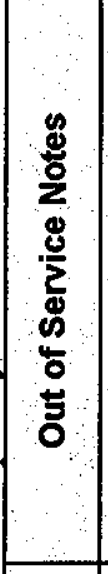 & 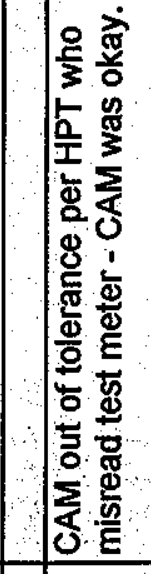 & $\underline{\mathbf{s}}$ & 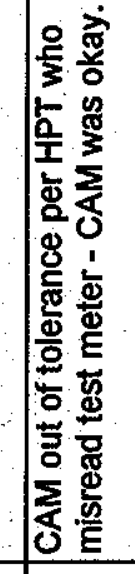 & 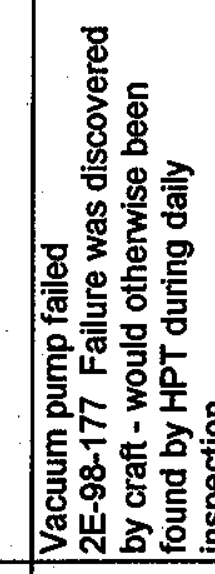 & 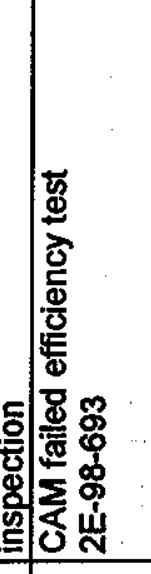 & 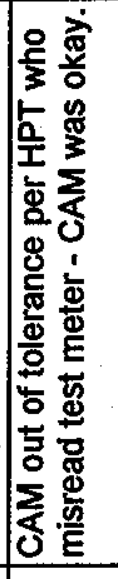 & 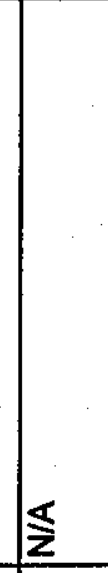 & $\underline{\mathbf{z}}$ & $\$$ & 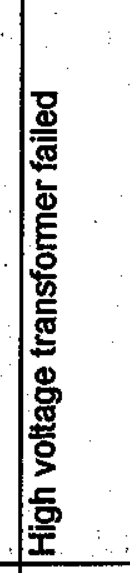 & 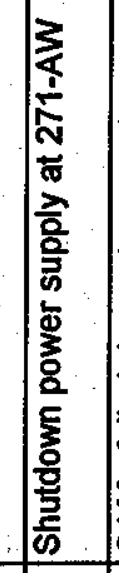 & 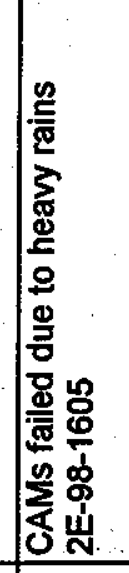 & 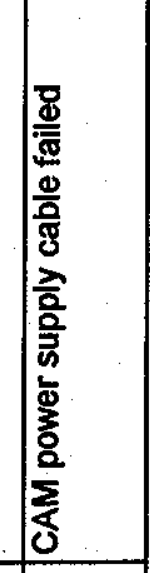 \\
\hline 总 & 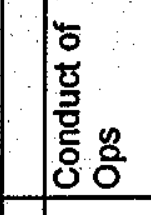 & $\Sigma$ & 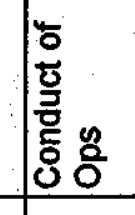 & 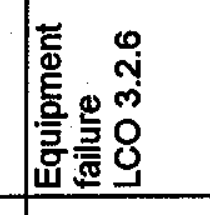 & 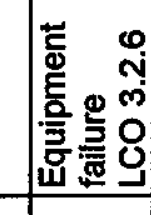 & 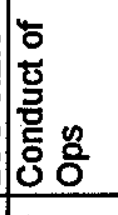 & $\frac{\$}{z}$ & $\Sigma$ & 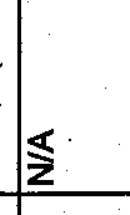 & 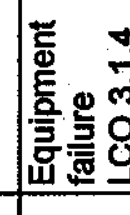 & 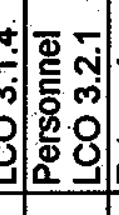 & 焉 & 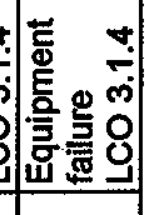 \\
\hline 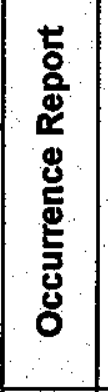 & 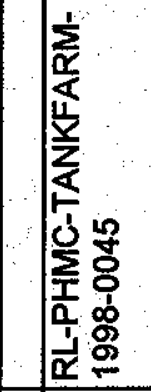 & 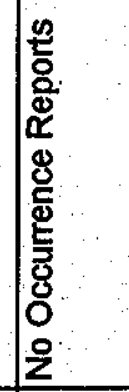 & 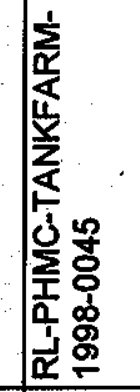 & 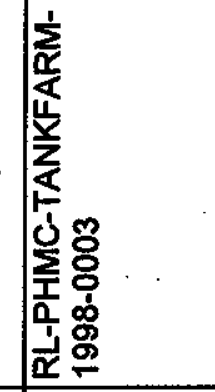 & 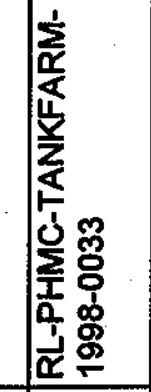 & 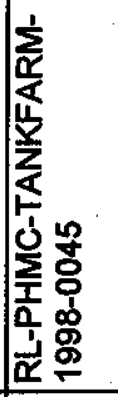 & 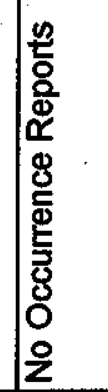 & 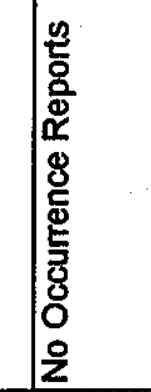 & 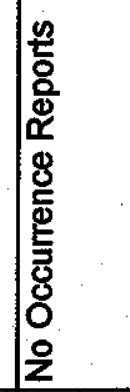 & 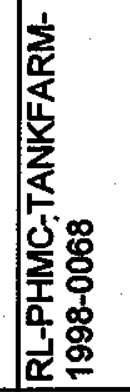 & 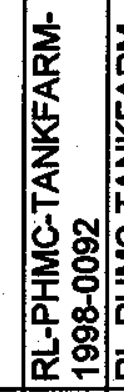 & 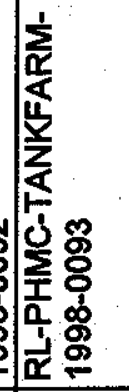 & 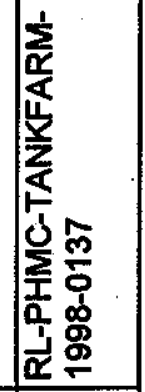 \\
\hline 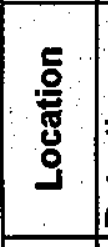 & 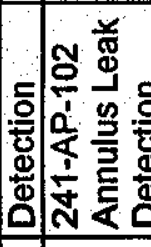 & 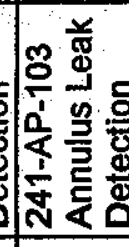 & 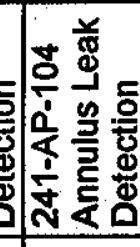 & 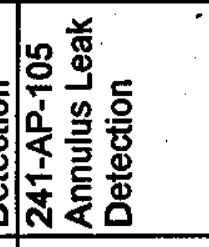 & 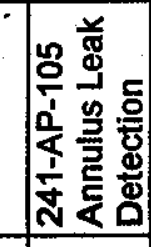 & 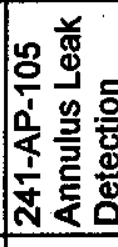 & 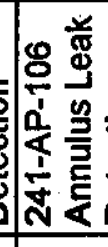 & 噌 & 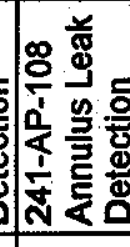 & 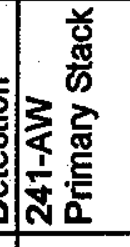 & 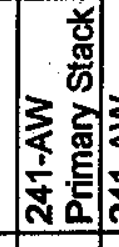 & 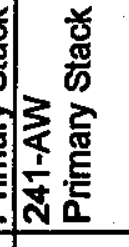 & 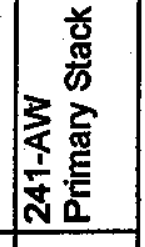 \\
\hline & 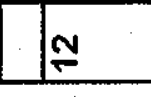 & m & \pm & px & & & & $F$ & $\stackrel{\infty}{-}$ & of & & & \\
\hline
\end{tabular}




\begin{tabular}{|c|c|c|c|c|c|c|c|c|c|c|c|c|c|c|}
\hline 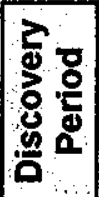 & 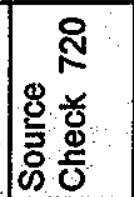 & \begin{tabular}{l}
\multirow{2}{*}{} \\
$\stackrel{5}{\mathbf{I}}$ \\
\end{tabular} & 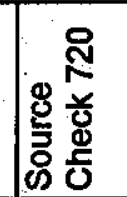 & $\mid \begin{array}{l}\mathbf{N} \\
\frac{n}{2}\end{array}$ & $\frac{\widehat{K}}{\mathbf{z}}$ & 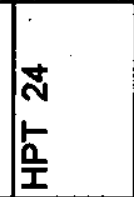 & & 尽 & 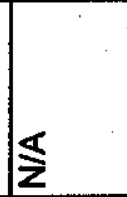 & $\leqslant$ & $\begin{array}{l}0 \\
0 \\
0\end{array}$ & $\begin{array}{l}\infty \\
0 . \\
0\end{array}$ & 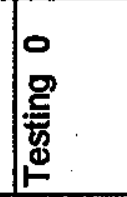 & $\begin{array}{l}0 \\
0 \\
0\end{array}$ \\
\hline 造 & 足 & & 胥 & $\frac{n}{\stackrel{n}{N}}$ & $\leqslant$ & $\frac{n}{\grave{n}}$ & 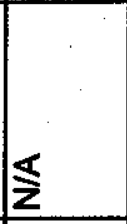 & 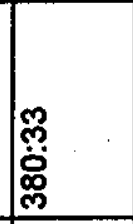 & $\leqslant$ & $\frac{\$}{z}$ & 㝦 & 웋 & 둥 & 胥 \\
\hline 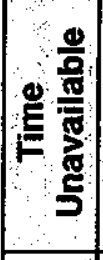 & 悹 & (ิ) & 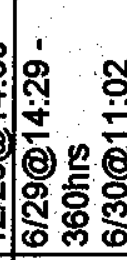 & 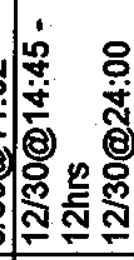 & $\frac{1}{z}$ & 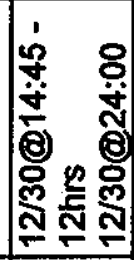 & $\frac{1}{z}$ & 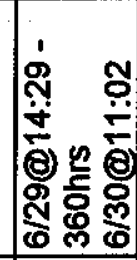 & $\frac{5}{2}$ & 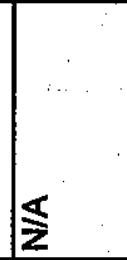 & 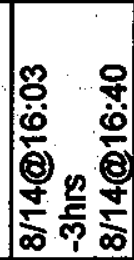 & 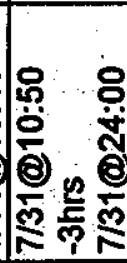 & 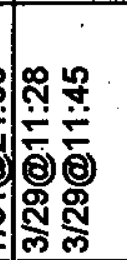 & 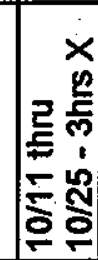 \\
\hline 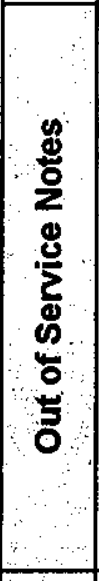 & 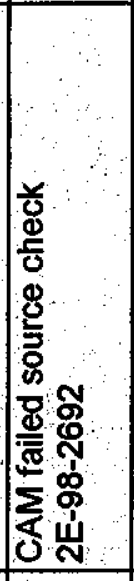 & 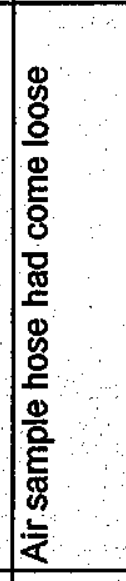 & 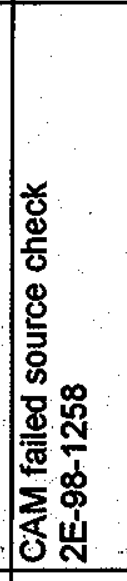 & 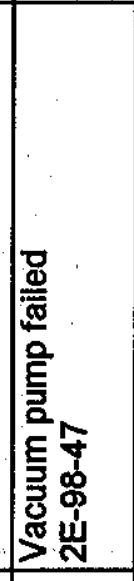 & $\leqslant$ & 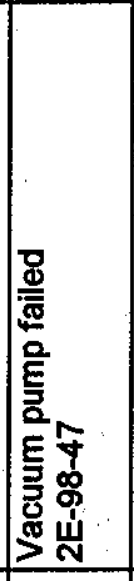 & $\leqslant$ & 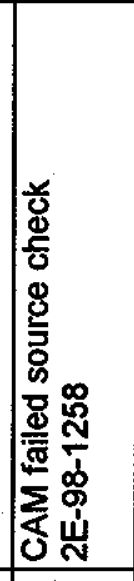 & 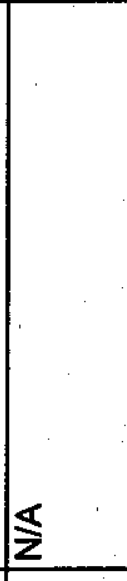 & $\$$ & 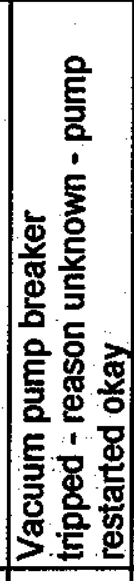 & 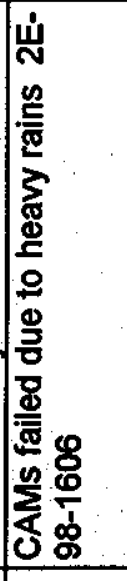 & 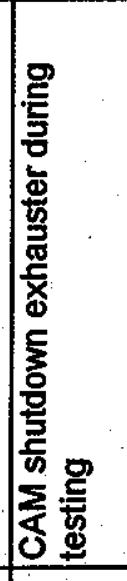 & 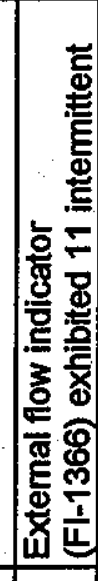 \\
\hline 늘 원 & 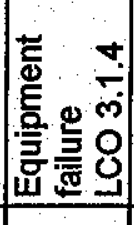 & 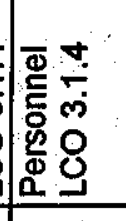 & 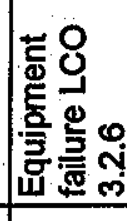 & 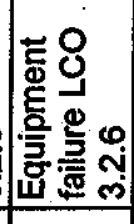 & $\frac{5}{z}$ & 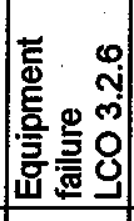 & $\leqslant$ & 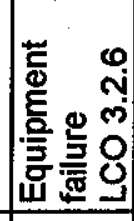 & 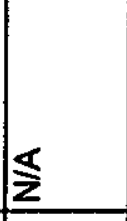 & 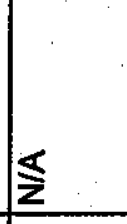 & 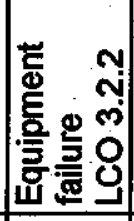 & 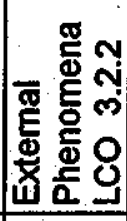 & 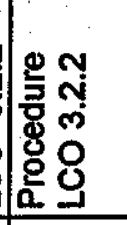 & 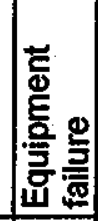 \\
\hline 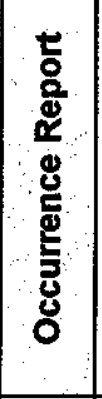 & 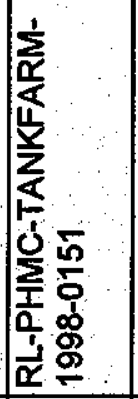 & 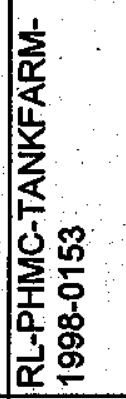 & 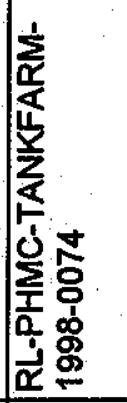 & 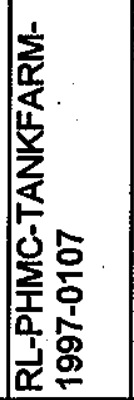 & 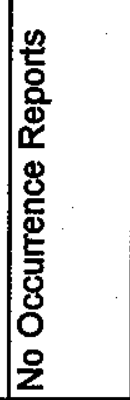 & 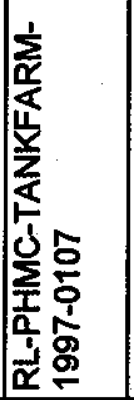 & 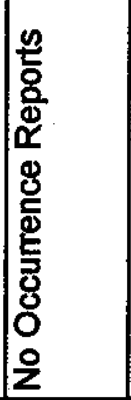 & 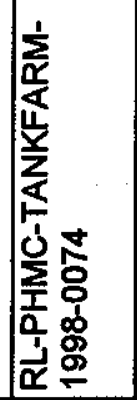 & 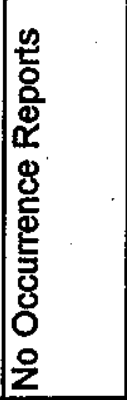 & 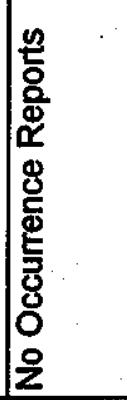 & 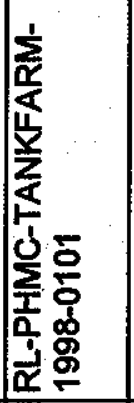 & 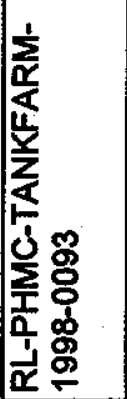 & 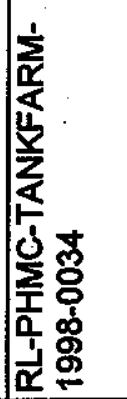 & 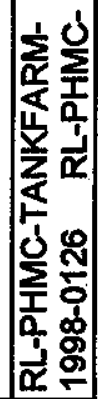 \\
\hline 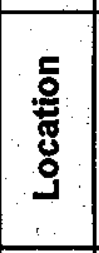 & 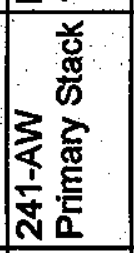 & 惫 & 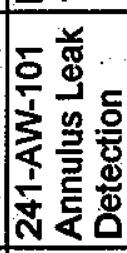 & 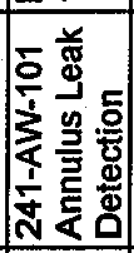 & 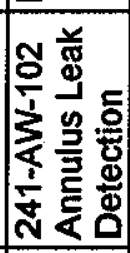 & 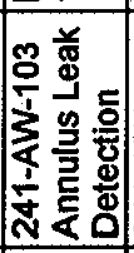 & 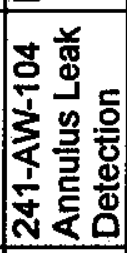 & 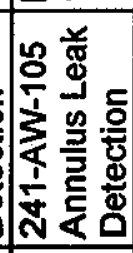 & 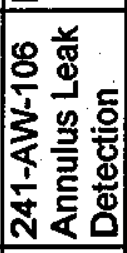 & 盖 & 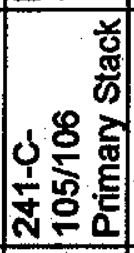 & 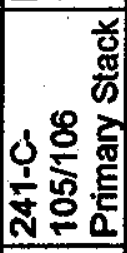 & 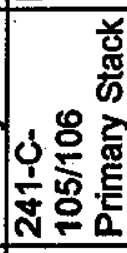 & 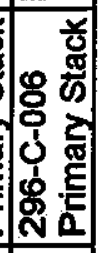 \\
\hline & & & R & & & |⿰冫 & $\mathbb{N}$ & I & నึ & $\mathscr{N}$ & $\sqrt{N}$ & & & \\
\hline
\end{tabular}




\begin{tabular}{|c|c|c|c|c|c|c|c|c|c|c|c|c|c|}
\hline 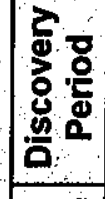 & & 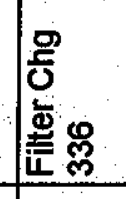 & $\begin{array}{l}0 \\
0 \\
0\end{array}$ & 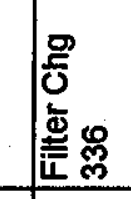 & $\begin{array}{l}0 \\
0 \\
0 \\
0\end{array}$ & 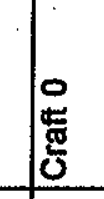 & $\begin{array}{l}0 \\
0 \\
0 \\
0\end{array}$ & 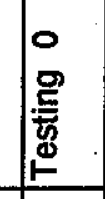 & $\leqslant$. & 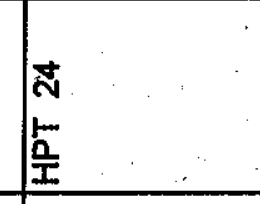 & 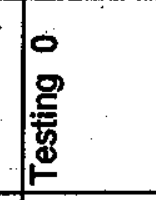 & 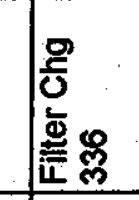 & $\begin{array}{l}0 \\
0 \\
0 \\
0\end{array}$ \\
\hline 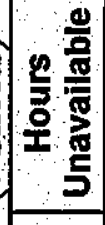 & & 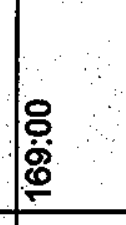 & 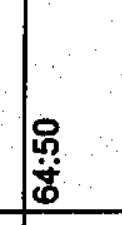 & 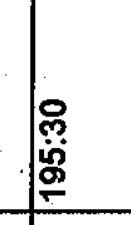 & 递 & 串 & 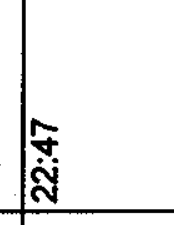 & 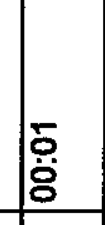 & $\Sigma$ & 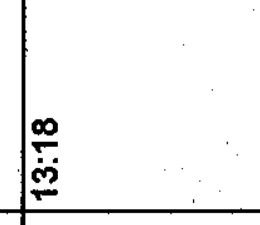 & ָิ๊ & 浐 & 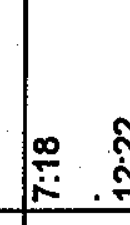 \\
\hline 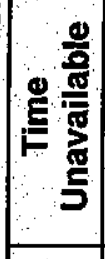 & $=$ & 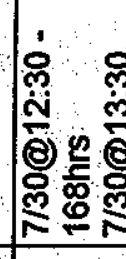 & : & 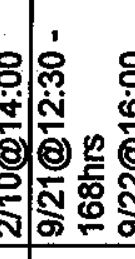 & ה̂: & $\frac{1}{4}$ & 离 & 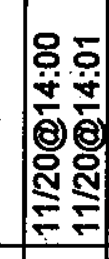 & s. & 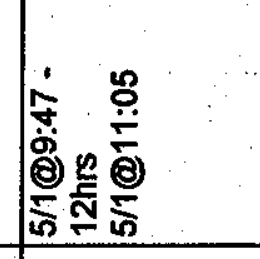 & 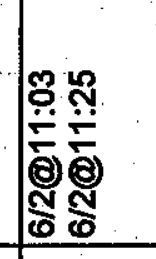 & 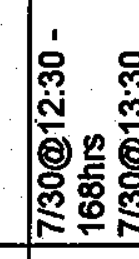 & : \\
\hline 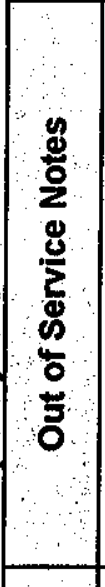 & 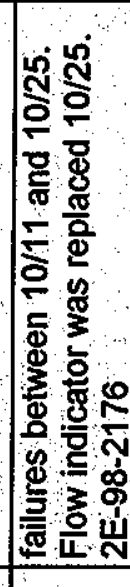 & 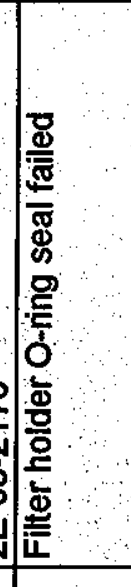 & 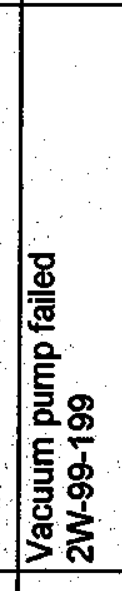 & 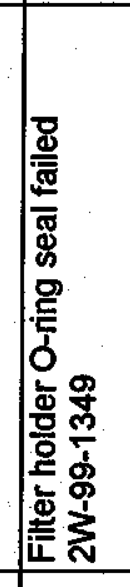 & 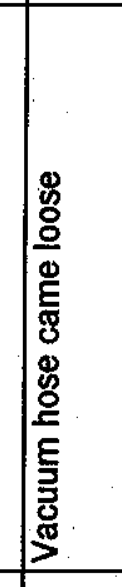 & 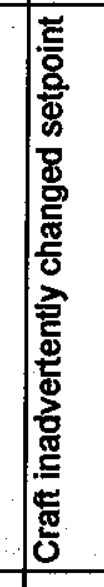 & 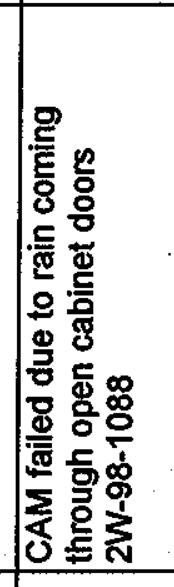 & 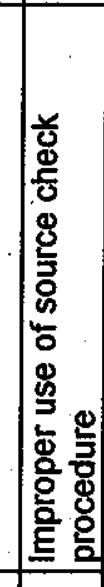 & 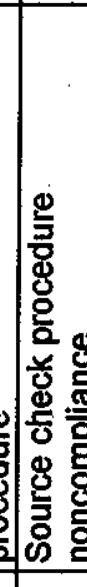 & 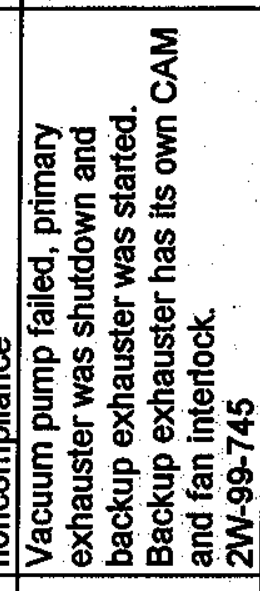 & 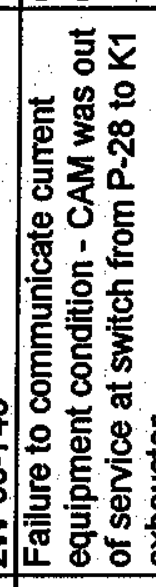 & 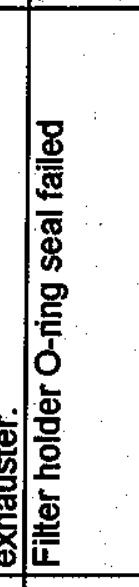 & 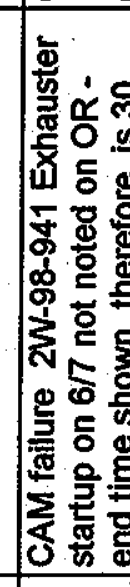 \\
\hline 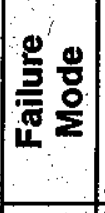 & •. & 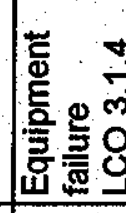 & 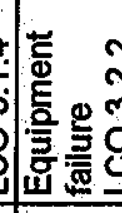 & 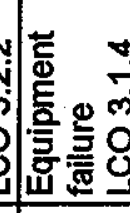 & 蒿 & : & 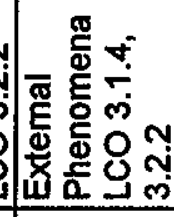 & 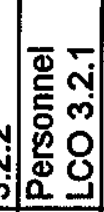 & 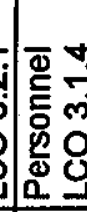 & 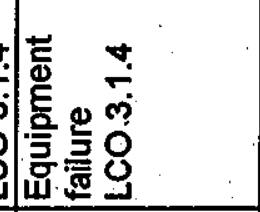 & 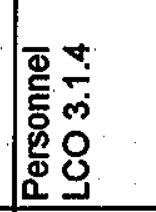 & 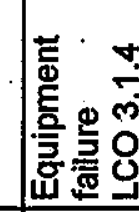 & : \\
\hline 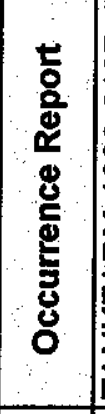 & 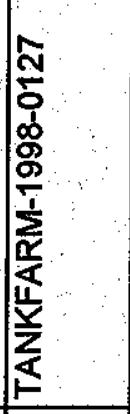 & 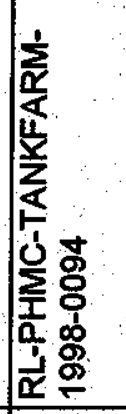 & 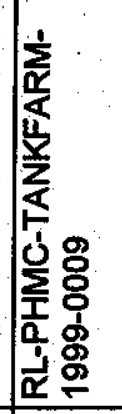 & 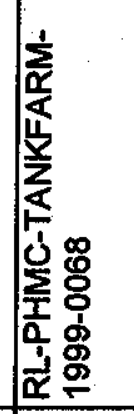 & 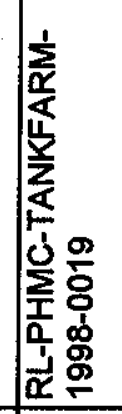 & 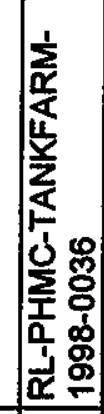 & 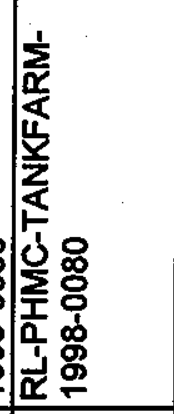 & 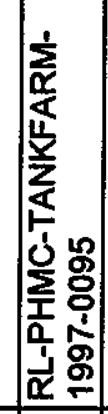 & 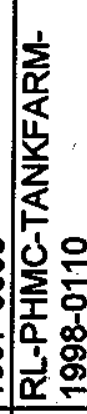 & 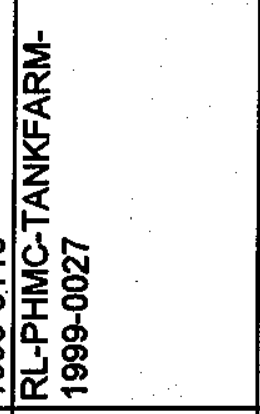 & 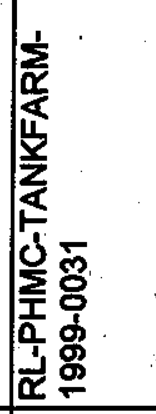 & 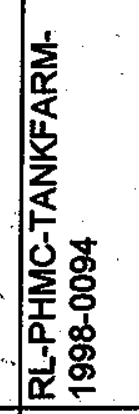 & 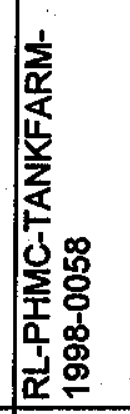 \\
\hline ذّ & & 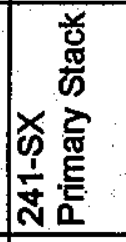 & 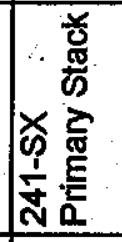 & 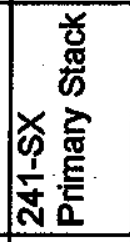 & 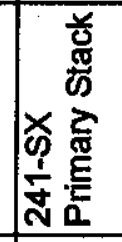 & 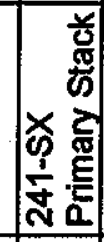 & 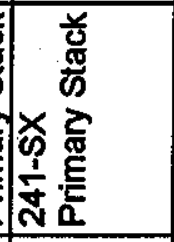 & 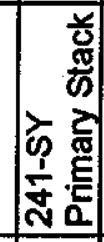 & 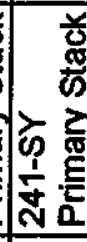 & 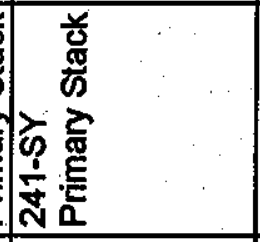 & 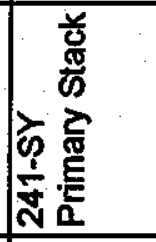 & 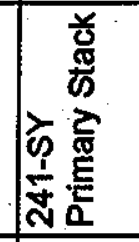 & 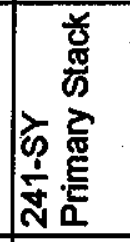 \\
\hline & & 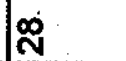 & & & & & & |ล & & & & & \\
\hline
\end{tabular}




\begin{tabular}{|c|c|c|c|c|c|c|c|c|c|c|c|c|}
\hline 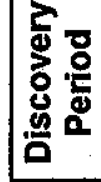 & & $\frac{\pi}{z}$ & $\underline{\Sigma}$ & $\underline{\mathbf{s}}$ & $\S$ & 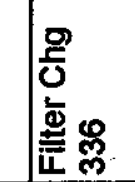 & $\mid \begin{array}{l}0 \\
0 \\
0 \\
0\end{array}$ & 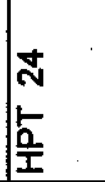 & 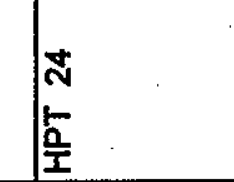 & $\begin{array}{l}0 \\
\check{0} \\
0\end{array}$ & 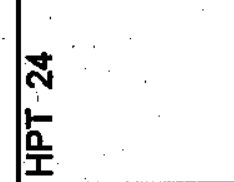 & $\begin{array}{l}\text { 竎 } \\
\text { 点 } \\
\text { 年 }\end{array}$ \\
\hline 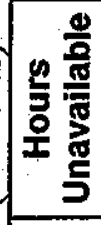 & & $\underline{\mathbf{z}}$ & $\underline{\mathbf{z}}$ & $\underline{\Sigma}$ & $\underline{\mathbf{z}}$ & ס̊. & 웅 & 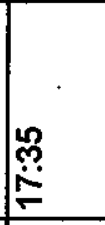 & 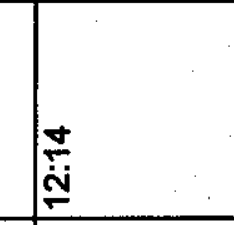 & 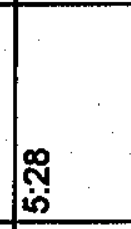 & 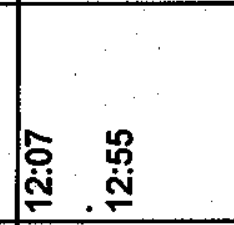 & 总 \\
\hline 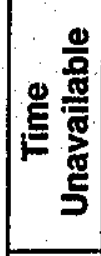 & 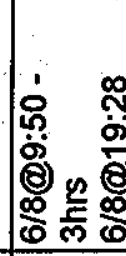 & : & $\underline{\mathbf{s}}$ & $\mathbf{\Sigma}$ & $\underline{\mathbf{s}}$ & 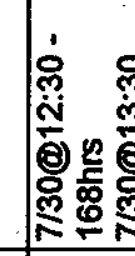 & 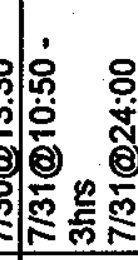 & 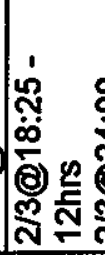 & 竎 & 递 & 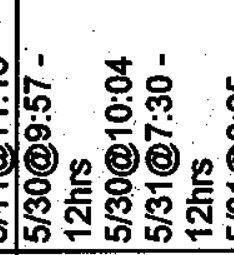 & 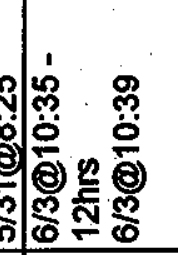 \\
\hline 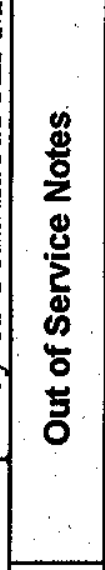 & 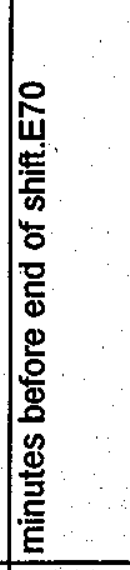 & $\underline{\mathbf{z}}$ & $\underline{\mathbf{z}}$ & 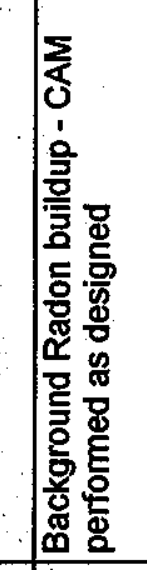 & $\S$ & 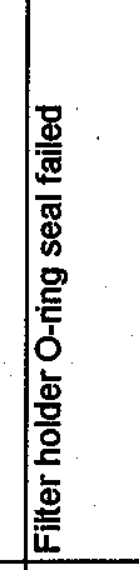 & 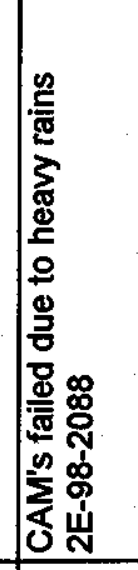 & 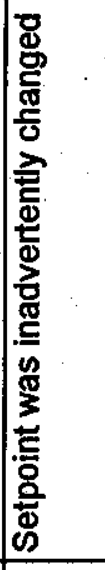 & 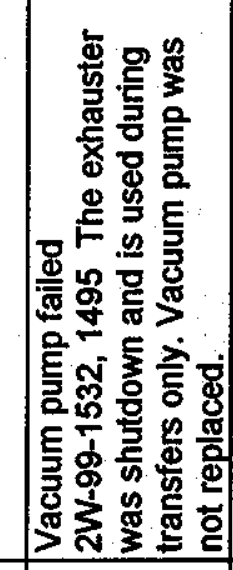 & 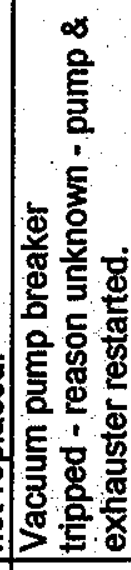 & 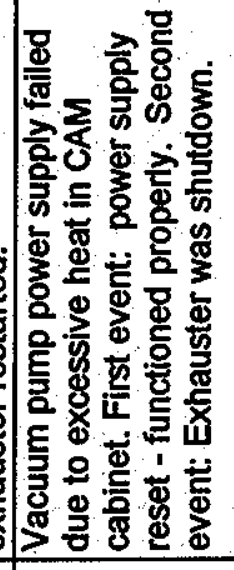 & 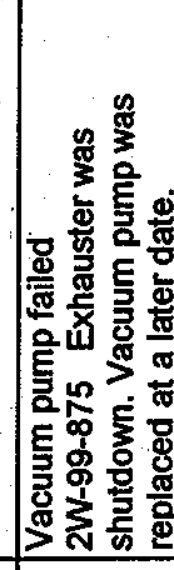 \\
\hline 獄 & & $\underline{\mathbf{s}}$ & $\underline{\Sigma}$ & 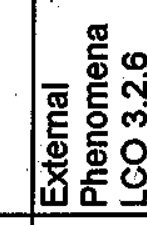 & : & 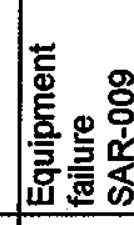 & 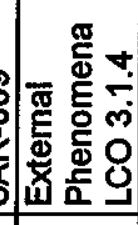 & 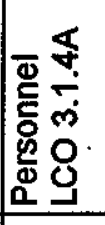 & 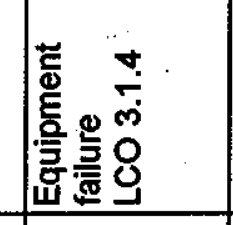 & 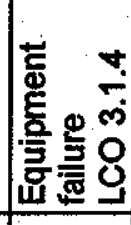 & 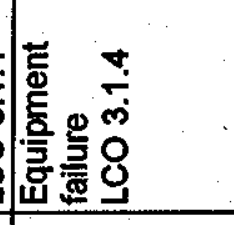 & 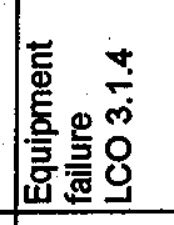 \\
\hline 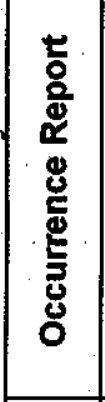 & & 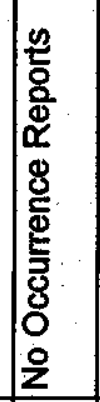 & 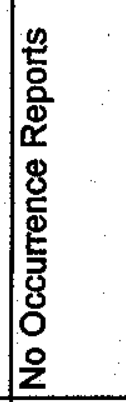 & 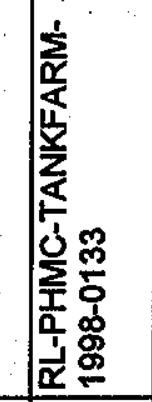 & 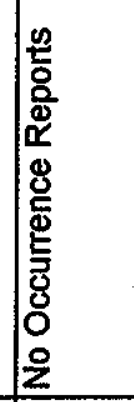 & 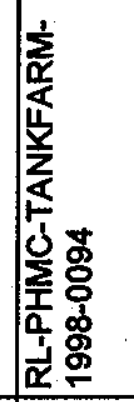 & 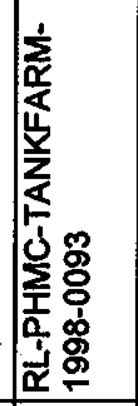 & 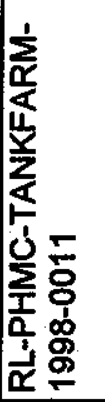 & 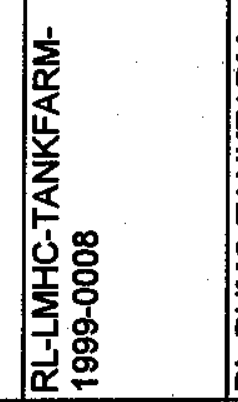 & 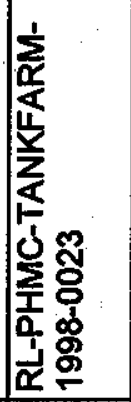 & 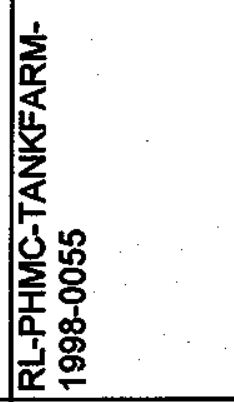 & 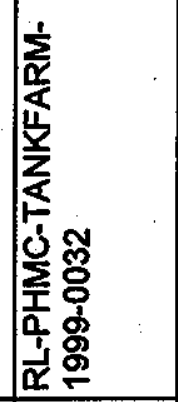 \\
\hline & & 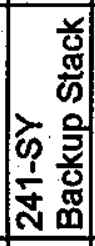 & 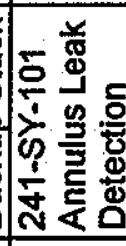 & 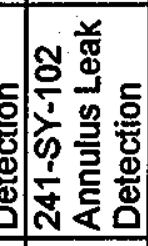 & 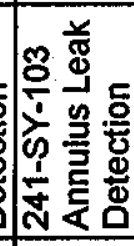 & 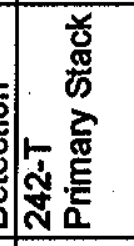 & 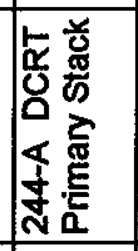 & 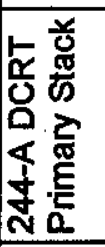 & 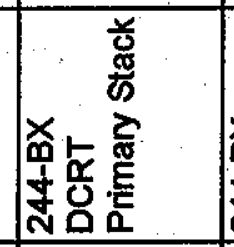 & 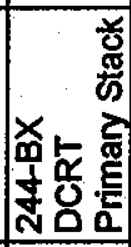 & 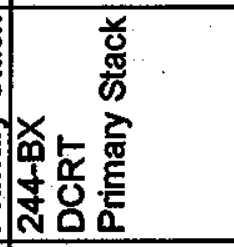 & 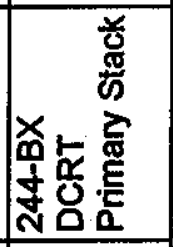 \\
\hline & & 足 & $\bar{m}$ & N్ల & 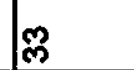 & |ूे & $\omega_{0}$ & & $1 \%$ & & & \\
\hline
\end{tabular}




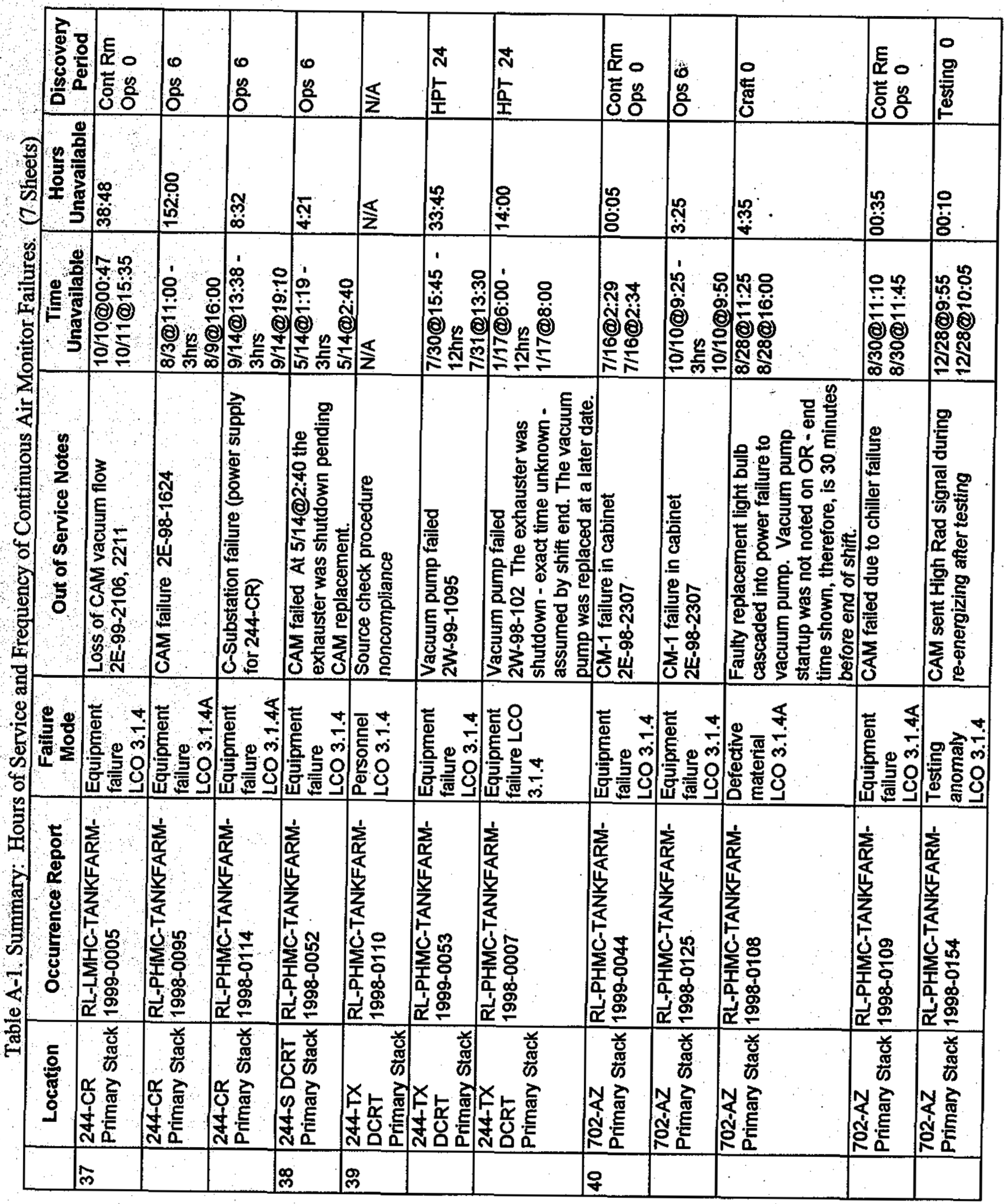




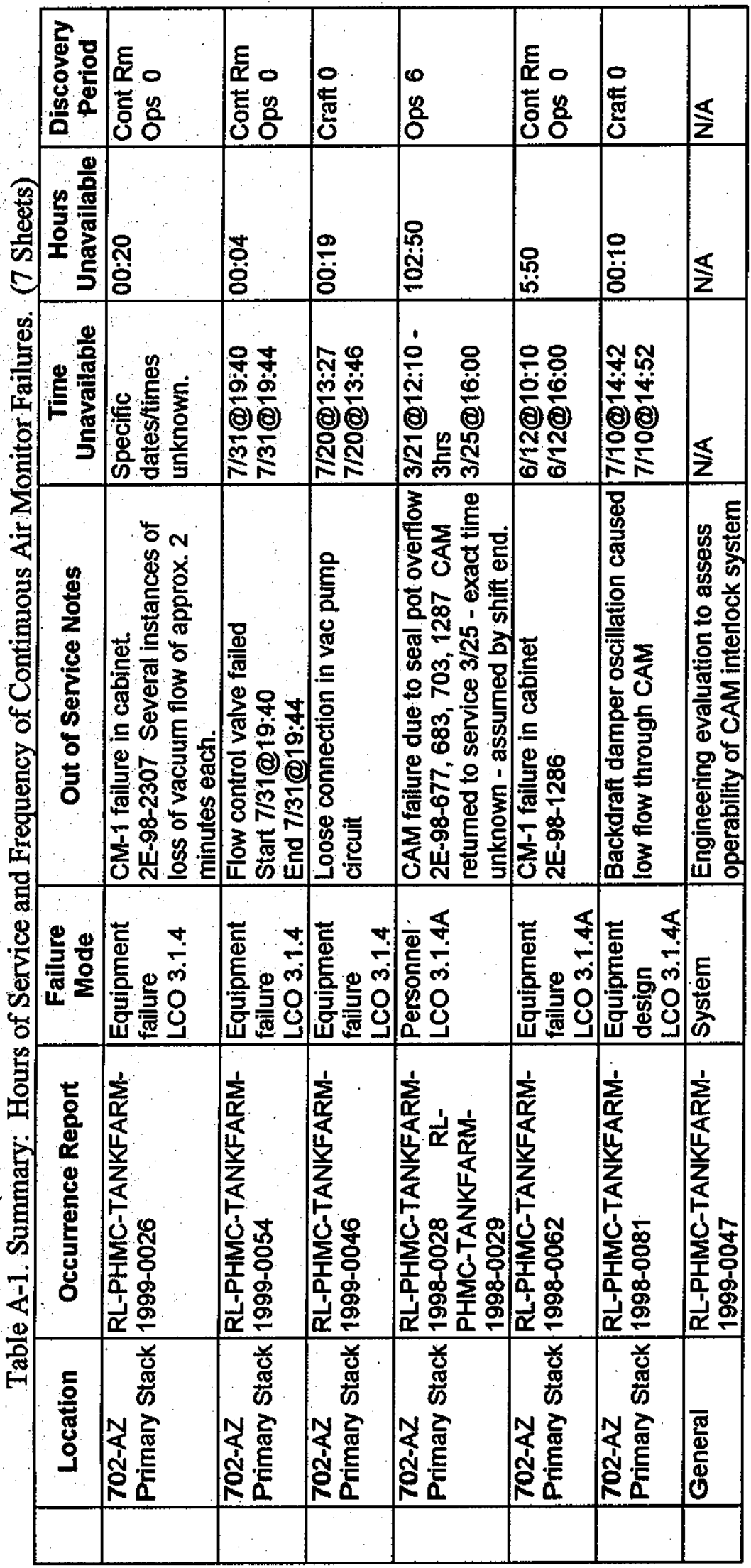


RPP-5453 REV 0

APPENDIX B

CONTINUOUS AIR MONITOR AVAILABILITY ANALYSIS

B-i 
RPP-5453 REV 0

This page intentionally left blank.

B-ii 


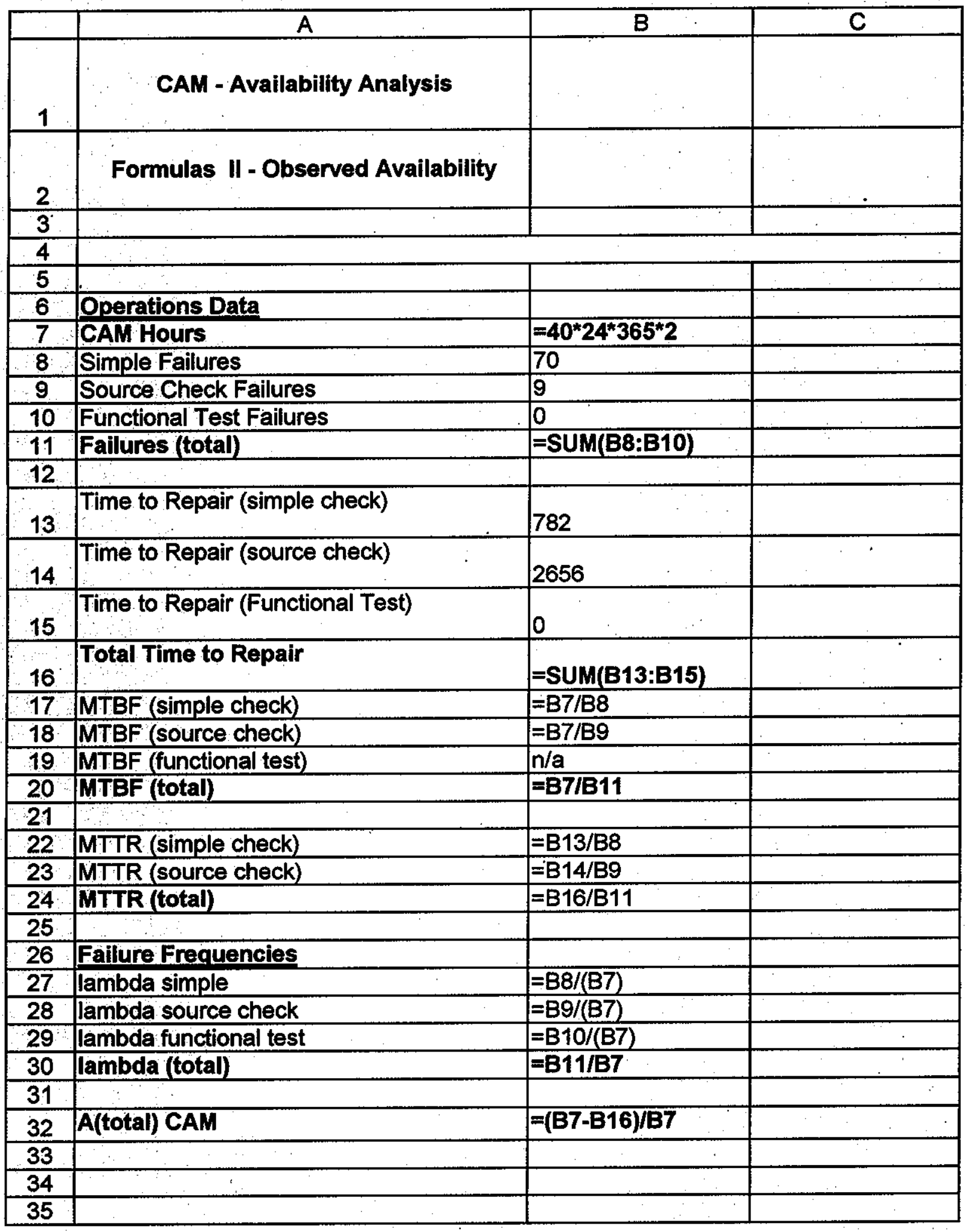




\begin{tabular}{|c|c|c|c|}
\hline & $\begin{array}{r}\mathrm{A} \\
\end{array}$ & $B$ & $\mathrm{C}$ \\
\hline 1 & Spreadsheet Formulas & & \\
\hline 2 & Calculated Availability & & \\
\hline 3 & & & \\
\hline 4 & & & \\
\hline 5 & & & \\
\hline 6 & Operations Data & & \\
\hline 7 & CAM Hours & $=2 * 40^{*} 365^{*} 24$ & \\
\hline 8 & Simple Failures & 70 & \\
\hline 9 & Source Check Failures & 9 & \\
\hline 10 & Functional Test Failures & 1 & \\
\hline 11 & Time to Repair (simple check) & 782 & \\
\hline 12 & Time to Repair (source check) & 2656 & \\
\hline 13 & Time to Repair (Functional Test) & 0 & \\
\hline 14 & MTBF (simple check) & $=\mathrm{B} 7 / \mathrm{B} 8$ & \\
\hline 15 & MTBF (source check) & $=\mathrm{B} 7 / \mathrm{B} 9$ & \\
\hline 16 & MTBF (functional test) & $=\mathrm{B} 7 / \mathrm{B} 10$ & \\
\hline 17 & MTTR (simple check) & $=\mathrm{B} 11 / \mathrm{B} 8$ & \\
\hline 18 & MTTR (source check) & $=\mathrm{B} 12 / \mathrm{B} 9$ & \\
\hline 19 & & & \\
\hline 20 & Failure Frequencies & & \\
\hline 21 & lambda simple & $=\mathrm{B} 8 /(\mathrm{B} 7)$ & \\
\hline 22 & lambda source check & $=\mathrm{B} 9 /(\mathrm{B} 7)$ & \\
\hline 23 & lambda functional test & $=\mathrm{B} 10 /(\mathrm{B} 7)$ & \\
\hline 24 & & & \\
\hline 25 & Surveillance Frequencies (hours) & & \\
\hline 26 & t simple & 24 & $\therefore$ \\
\hline 27 & t source check & 720 & \\
\hline 28 & $t$ functional test & 2208 & \\
\hline 29 & & & \\
\hline 30 & Availability Based on Surveillance & & \\
\hline 31 & A(sur) simple & $=1-0.5^{*}\left(\mathrm{~B} 21^{*} \mathrm{~B} 26\right)$ & \\
\hline 32 & $A$ (sur) functional & $=1-0.5^{*}\left(B 22^{*} B 27\right)$ & \\
\hline 33 & $A($ sur) interlock & $=1-0.5^{*}\left(\mathrm{~B} 23^{*} \mathrm{~B} 28\right)$ & \\
\hline 34 & A(sur) CAM & $=\mathrm{B} 31^{*} \mathrm{~B} 32^{*} \mathrm{~B} 33$ & \\
\hline 35 & & & \\
\hline 36 & Availability Based on Time to Repair & & \\
\hline 37 & $A$ (repair) alarm & $=(B 14 /(B 14+B 17))$ & \\
\hline 38 & A(total) CAM & $=\mathrm{B} 37^{*} \mathrm{~B} 33^{*} \mathrm{~B} 32$ & \\
\hline 39 & & & \\
\hline 40 & & & \\
\hline 41 & & & \\
\hline
\end{tabular}


RPP-5453 REV 0

APPENDIX C

AVAILABILITY ANALYSIS CASES FOR RPP CONTINUOUS AIR MONITOR INTERLOCK SYSTEMS 


\section{RPP-5453 REV 0}

This page intentionally left blank.

C-ii 


\section{CAM - Availability Analysis}

\section{Availability based on observation $=$ (Total CAM hours - Total Time to Repair $) /($ Total CAM hours)}

\section{Operations Data}

\section{CAM Hours}

Simple Failures

Source Check

Failures

Functional Test

Failures

Failures (total)

Time to Repair

(simple check)

Time to Repair

(source check)

Time to Repair

(Functional Test)

Total Time to Repair $3.44 E+03$

MTBF (simple check) 1.001E+04

MTBF (source check) 7.787E+04

MTBF (functional

test)

MTBF (total)

MTTR (simple check) 1.117E+01

MTTR (source check) 2.951E+02

Failure Frequencies

$$
\text { lambda simple }
$$

lambda source check

lambda functional

test

lambda (total)

A(total) CAM

$7.82 \mathrm{E}+02$

$2.66 \mathrm{E}+03$

$0.00 E+00$

$\mathrm{n} / \mathrm{a}$

8.871E+03

9.99E-05

1.28E-05

$0.00 E+00$

1.13E-04
$7.008 E+05$
$7.00 E+01$
$9.00 E+00$

$0.00 E+00$ (there have been no observed functional test failures)

7.90E+01

0.995 (availability based on fraction of time observed available) 


\section{CAM - Availability Analysis}

(1. Failures detected by simple check, 2. Failures detected by source check, 3 . Failure detected by system function check only)

Case 1 - CAM Availability - Based on Daily Simple Check, 30-Day Source Check, and a 92Day Functional Test

\section{Operations Data}

CAM Hours

Simple Failures

Source Check Failures

Functional Test

Failures

Time to Repair (simple check)

Time to Repair (source check)

Time to Repair

(Functional Test)

MTBF (simple check)

MTBF (source check)

MTBF (functional test)

MTTR (simple check)

MTTR (source check)

\section{Failure Frequencies}

\begin{tabular}{ll}
\hline lambda simple & $9.99 \mathrm{E}-05$ \\
lambda source check & $: \begin{array}{l}1.28 \mathrm{E}-05 \\
\text { lambda functional test }\end{array}$ \\
\hline
\end{tabular}

Surveillance Frequencies (hours)

t simple

t source check

$t$ functional test
$7.008 \mathrm{E}+05$

$7.00 \mathrm{E}+01$

$9.00 \mathrm{E}+00$

$1.00 \mathrm{E}+00$ (one is conservative relative to experience of zero)

$7.82 E+02$

$2.66 \mathrm{E}+03$

$0.00 E+00$

$1.001 \mathrm{E}+04$

7.787E+04

$7.008 \mathrm{E}+05$

1.117E+01

2.951E+02

9.99E-05

1.43E-06

24 (simple surveillance check frequency in hours)

720 (CAM source check frequency in hours)

2208 (system functional test surveillance frequency in hours)

Availability Based on Surveillance

A(sur) simple $\quad 9.988 \mathrm{E}-01$

$\mathrm{A}($ sur) functional $\quad 9.954 \mathrm{E}-01$

$\mathrm{A}$ (sur) interlock $\quad 9.984 \mathrm{E}-01$

A(sur) CAM

0.993 (availability if determined by surveillance frequency) 


\section{CAM - Availability Analysis}

(Failures detected by simple check, source check, and system function check are alarmed)

\section{Case 2 - CAM Availability - If All CAM Failures Were Alarmed to a Continuously Manned Station with Immediate Response}

\begin{tabular}{ll} 
Operations Data & \\
\hline CAM Hours & $7.008 \mathrm{E}+05$ \\
Simple Failures & $7.00 \mathrm{E}+01$ \\
Source Check Failures & $9.00 \mathrm{E}+00$ \\
\hline Functional Test & $1.00 \mathrm{E}+00$ (one is conservative relative to experience of zero) \\
$\begin{array}{l}\text { Failures } \\
\text { Time to Repair (simple }\end{array}$ & $7.82 \mathrm{E}+02$ \\
check) & \\
\hline Time to Repair (source & $2.66 \mathrm{E}+03$ \\
check) & \\
\hline Time to Repair & $0.00 \mathrm{E}+00$ \\
(Functional Test) & \\
MTBF (simple check) & $1.001 \mathrm{E}+04$ \\
MTBF (source check) & $7.787 \mathrm{E}+04$ \\
MTBF (functional test) & $7.008 \mathrm{E}+05$ \\
MTTR (simple check) & $1.117 \mathrm{E}+01$ \\
MTTR (source check) & $2.951 \mathrm{E}+02$
\end{tabular}

\section{Failure Frequencies}

$\begin{array}{ll}\text { lambda simple } & 9.99 \mathrm{E}-05 \\ \text { lambda source check } & 1.28 \mathrm{E}-05 \\ & 1.43 \mathrm{E}-06\end{array}$

lambda functional test $\quad 1.43 \mathrm{E}-06$

\section{Surveillance Frequencies (hours)}
t simple
0.5 (failure alarm with $30-\mathrm{min}$ response)
t source check
0.5 (failure alarm with $30-\mathrm{min}$ response)
t functional test
0.5 (failure alarm with $30-\mathrm{min}$ response)

Availability Based on Time to

\section{Repair}

A(repair) alarm

9.989E-01

$A$ (sur) functional

9.962E-01

A(sur) interlock

A(total) CAM 


\section{CAM - Availability Analysis}

(1. Failures detected by simple check, 2. Failures detected by source check, 3. Failure detected by system function check only)

\section{Case 3 - CAM Availability - Based on 92 Day Functional Test Only}

\section{Operations Data}

CAM Hours

Simple Failures

Source Check Failures

Functional Test

Failures

Time to Repair (simple check)

Time to Repair (source check)

Time to Repair

(Functional Test)

MTBF (simple check)

MTBF (source check)

MTBF (functional test)

MTTR (simple check)

MTTR (source check)

Failure Frequencies

lambda simple

'lambda source check

lambda functional test
7.008E+05

$7.00 E+01$

$9.00 E+00$

$1.00 \mathrm{E}+00$ (one is conservative relative to experience of zero)

$7.82 \mathrm{E}+02$

2.66E+03

$0.00 E+00$

$1.001 E+04$

7.787E+04

7.008E+05

1.117E+01

2.951E+02

9.99E-05

1.28E-05

1.43E-06

Surveillance Frequencies (hours)

t simple

t source check

t functional test
2208 (simple surveillance check frequency in hours) 2208 (CAM source check frequency in hours)

2208 (system functional test surveillance frequency in hours)

Availability Based on Surveillance

$A$ (sur) simple

8.897E-01

$A$ (sur) functional

9.858E-01

$A$ (sur) interlock

9.984E-01

A(sur) CAM

0.876 (availability determined by surveillance frequency) 
RPP-5453 REV 0

\section{APPENDIX D \\ COPY OF CONTRACTOR SURVEILLANCE REPORT RESPONSE S99-TOD-TF-042-FO1}

D-i 
RPP-5453 REV 0

This page intentionally left blank.

D-ii 
$(10,-5,582.2$

Surveillance Number: S99-TOD-TE-042-801

Date: September 2, 1999

Page 1 of 2 . . :

\section{CONTRACTOR SURVEILLANCE REPORT RESPONSE}

DOE-RL Author: B. J. Harp

Subject/Scope of Survellance:

Technical safety Requirements

Surveillance Results:

Safety class system operability requizements wexe not met for the ventilation stack continuous air monitor (CAM) intexiock system (LCO 3.1.4).

Requirement: DOE Order 5480.22 , "Technical Safety Requixements," Attachment i, Section II, Paragraphs. $2.4(\mathrm{~h})$ and 2.5 are implemented through HNE-DS-WM-TSR-006, "Tank Waste Remediation system . Technical Safety Requirements."

Backgroud: Provided on page 2

Discussion and Explanation:

DOR Q R scussion and Exolanation: The definition of operable-operability (HNE-SD-WM-TSR-006) specifies. that a system and all necessary attendant equipment shall be capable of performing the systems specified function. The functional requirement for the CAM. (HNF-SD-WM-SEL-040) specifies that the CAM must measure the sample flow stream and shutdown the exhauster within 10 minutes of exceeding a preset radiation level. Continued on page 2

Root Cause:

4B - Design Problem; inadequate or defective design

Corrective Actions:

1. Perform an engineering evaluation to provide reasonable assurance of operability with applicable compensatary actions (if needed) - Plant Engineering by $09 / 08 / 99$.

2: Perform an engineering evaluation to determine fajlure modes that af fect the intended safety function of the CAMs with regard to the interlock TSR and make necessary recommendations to correct any" deficiencies - Equipment Engineering by 11/15/99.

3. Analyze surveiliance frequency to determine adequacy - Safety Analysis by $11 / 15 / 99$. 4. After completion of the engineer evaluation, develop a corrective action plan for implementation - Tank Farm Facility Operations by 12/27/99.

Planned Completion Date: December 27, 1999

Responsible Contractor Individual: c. DiFrango

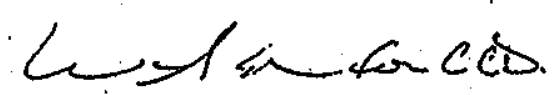




\section{$\cdot$ CONTRACTOR SURVEILLANCE REPORT RESPONSE (CONTINUATION)}

SURVEILLANCE RESULTS: - continued

Background: The computer automazed survelllance system (CASs) provided remote alarms, at a continuously manned location, that detected equipment failures of the CAM interlock system. The cass was rexoved from service based on the results of unreviewed safety question determinations (USQDS) TE-98-0829 and TE-99-0142. These USQDs provided tho justification for eliminating CASS.

The TSR bases section for LCO 3.1.: refexences the calculation note that analyzed a HEPA filter faflure accident and subsequent radionuclide release with a 10 minute duration. The consequence of the 10 minute release was within acceptance guidelines. Based on this analyses, a 10 minute ventilation system shutdown time for the. stack CAM intexlock system was established.

DISCUSSTON ANO EXLANATYON: - continued .

DQE Discussion and Explanation: - continued

Contrary to this functional requirement; the CAM is not capable of shutting down the exhauster within 10 minutes when a CAM equipment failure, e.g. vacuum pump, occurs. If an equipment fallure occurred, the CAM could operate for up to 6 hours in an inoperable status because the CAM is not interlocked to shutdown the exhauster and alarms are not monitored continuousiy: Therefore, the ventilation stack caM interlock. system does not meet operability requirements because the system 10 minute shutdown functional requirement cannot be met upon failure of a system safety class component.

LMHC Discussion and Explanation: HNE-SD-WM-SEL-040, Section 6.1 .1 .3 states the "The CAM shall activate an interlock to shut down the exhauster within 20 minutes of detecting a radiation level that exceeds the preset level. On CAM fallure, the. monitors must actuate an alarm and/or an interlock to shut down the exhaust system." These two-sentences differentiate between CAM activation due to detecting radiation levels and CAM failuxes. The interlock and 10 minute requirements are applicable to CAM activation. CAM systern faflure is detected by remote monitors located in instrument buildings. This is considered acceptable because of the low probability of an event occurring concurrent with a supporting system failuxe. 
RPP-5453 REV 0

APPENDIX E CHECKLIST FOR TECHNICAL PEER REVIEW

E-i 
RPP-5453 REV 0

This page intentionally left blank

E-ii 


\section{CHECKLIST FOR TECHNICAL PEER REVIEW}

Document Reviewed: RPP-5453, "Evaluation of CAM Failure Modes and Analysis of Surveillance Frequency to Determine Adequacy"

Scope of Review: Check data and conclusions in text, and calculations shown in Appendices B and C of RPP-5453.

Yes No NA

[ ] [ ] [X] Previous reviews are complete and cover the analysis, up to the scope of this review, with no gaps. (No previous reviews exist.)

$[\mathrm{X}][][]$ Problem is completely defined.

[ ] [ ] [X] Accident scenarios are developed in a clear and logical manner. (Accident scenarios do not apply for RPP-5453.)

[X] [ ] [] Necessary assumptions are explicitly stated and supported.

$[\mathrm{X}][][] \quad$ Computer codes and data files are documented. (Excel spreadsheet)

[X] [ ] [ ] Data used in calculations are explicitly stated.

[X] [ ] [] Data were checked for consistency with original source information as applicable. (i.e., Appendix A, per JMG)

[X] [ ] [ ] Mathematical derivations were checked including dimensional consistency of results.

[ X] [ ] [] Models are appropriate and were used within their established range of validity or adequate justification was provided for use outside their established range of validity.

$[\mathrm{X}][\mathrm{]}$ [] Spreadsheet results and all hand calculations were verified.

[X] [ ] [ ] Software input (i.e., spreadsheet input) is correct and consistent with the document reviewed.

[ X] [ ] [ ] : Software output (i.e., spreadsheet results) is consistent with the input and with the results reported in the document reviewed.

[X] [ ] [ ] Limits/criteria/guidelines applied to the analysis results are appropriate and referenced. Limits/criteria/guidelines were checked against references. (per NUREG/CR-2300.)

[X] [ ] [ ] Safety margins are consistent with good engineering practices. (The purpose of RPP-5453 is to assess reliability of CAMs, which relates to safety margins.)

$[\mathrm{X}][][] \quad$ Conclusions are consistent with analytical results and applicable limits.

$[X][] \quad[] \quad$ Results and conclusions address all points in the purpose.

[ ] [X] The document was prepared in accordance with HNF-2353, Section 4.3, Attachment B, "Calculation Note Format and Preparation Instructions".

[ $\mathbf{X}]$ [ ] [ ] Concurrence (RPP-5453 is NOT a calcnote and uses a different format.)

Original signed by E. C. Heubach, II

Reviewer (Printed Name and Signature) $01 / 03 / 00$

Date 
RPP-5453

\section{DISTRIBUTION}

Number of copies

\section{ONSITE}

3

CH2M Hill

R. J. Cash

R1-44

G. L. Jones

R1-44

D. H. Shuford

R1-56

1

Duke Engineering, \& Services, Inc.

E. C. Heubach

R1-43

1

Fluor Technical Services

E. O. Thorne

R1-56

1

G\&P Consulting, Inc.

J. M. Grigsby

R1-44

1

$\underline{\text { MSI }}$

J. Young

R1-44

Distr-1 